Accepted on February 11, 2013, for the publication of ApJ

\title{
High-resolution Imaging of the Gegenschein and the Geometric Albedo of Interplanetary Dust
}

\author{
Masateru ISHIGURO \\ Astronomy Program, Department of Physics and Astronomy, Seoul National University, \\ 599 Gwanak-ro, Gwanak-gu, Seoul 151-742, Republic of Korea \\ ishiguro@astro.snu.ac.kr \\ Hongu YANG \\ Astronomy Program, Department of Physics and Astronomy, Seoul National University, \\ 599 Gwanak-ro, Gwanak-gu, Seoul 151-742, Republic of Korea \\ Fumihiko UsuI \\ Department of Infrared Astrophysics, Institute of Space and Astronautical Science (ISAS), JAXA, \\ 3-1-1 Yoshinodai, Chuo-ku, Sagamihara, Kanagawa 252-5210, Japan \\ Jeonghyun PYO \\ Korea Astronomy and Space Science Institute (KASI), Daejeon 305-348, Republic of Korea \\ Munetaka Ueno \\ Center for Space Science Technology, Institute of Space and Astronautical Science (ISAS), JAXA, \\ 3-1-1 Yoshinodai, Chuo-ku, Sagamihara, Kanagawa 252-5210, Japan \\ Takafumi Oотsubo \\ Astronomical Institute, Tohoku University, 6-3 Aramaki, Aoba-ku, Sendai 980-8578, Japan \\ Suk Minn KwON \\ Department of Science Education, Kangwon National University, \\ 192-1 Hyoja-dong, Kangwon-do, Chunchon 200-701, Republic of Korea \\ Tadashi MukAI \\ Department of Earth and Planetary Sciences, Kobe University, \\ 1-1 Rokkodai-cho, Nada-ku, Kobe 657-8501, Japan
}

\section{ABSTRACT}


We made optical observations of the Gegenschein using a liquid-nitrogen-cooled wide-field camera, Wide-field Imager of Zodiacal light with ARray Detector (WIZARD), between March 2003 and November 2006. We found a narrow brightness enhancement superimposed on the smooth gradient of the Gegenschein at the exact position of the antisolar point. Whereas the Gegenschein morphology changed according to the orbital motion of the Earth, the maximum brightness coincided with the antisolar direction throughout the year. We compared the observed morphology of the Gegenschein with those of models in which the spatial density of the interplanetary dust cloud was considered and found that the volume scattering phase function had a narrow backscattering enhancement. The morphology was reproducible with a spatial distribution model for infrared zodiacal emission. It is likely that the zero-phase peak (the so-called opposition effect) was caused by coherent backscattering and/or shadow-hiding effects on the rough surfaces of individual dust particles. These results suggest that big particles are responsible for both zodiacal light and zodiacal emission. Finally, we derived the geometric albedo of the smooth component of interplanetary dust, assuming big particles, and obtained a geometric albedo of $0.06 \pm 0.01$. The derived albedo is in accordance with collected dark micrometeorites and observed cometary dust particles. We concluded that chondritic particles are dominant near Earth space, supporting the recent theoretical study by dynamical simulation.

Subject headings: interplanetary medium — instrumentation: miscellaneous — comets: general — minor planets, asteroids

\section{Introduction}

The Gegenschein is a faint glow in the sky in the antisolar direction. Several hypotheses had been suggested to explain the Gegenschein: sunlight reflected by particles concentrated near the libration point in the Sun-Earth-particles system, emission from the Earth's gaseous tail, and sunlight scattered by the Earth's dust tail. After the detection of the Gegenschein at 1.86 AU from the Sun by the Pioneer 10 spacecraft (Weinberg et al. 1973; Hanner 1974), it has been widely accepted that the Gegenschein is a portion of zodiacal light enhanced by the backscattering of interplanetary dust particles. Intensive observations of the Gegenschein date back nearly half a century. It was observed by integrating the brightness in the field of view using photomultipliers attached to telescopes or telephotographic lenses (Tanabe 1965); Roosen 1970a; Dumont \& Sanchez 1975; Kwon et al. 2004). These authors observed the sky background within a certain interval on the sky $\left(1^{\circ}-5^{\circ}\right)$ or scanned the sky with large-field-of-view instruments. A volume scattering phase function of the interplanetary dust particles was deduced based on low-spatial-resolution data (Dumont \& Sanchez 1975; Leinert et al. 1976; Hong 1985; Lumme \& Bowell 1985; Lamy \& Perrin 1986). 
Modern observation techniques enable us to obtain snapshot images of the Gegenschein. James et al. (1997) were the first to used a cooled CCD camera attached to a fish-eye lens for the observation of zodiacal light and the Gegenschein, obtaining sufficient resolution (4.8 pixel $^{-1}$ ) images. Subsequently, a research group at Kobe University obtained images using a Peltier-cooled CCD camera (with a quantum efficiency of $10 \%$ at $0.44 \mu \mathrm{m}$ ) with an equatorial mounting and succeeded in detecting asteroidal dust bands on the Gegenschein (Ishiguro et al. 1999a (b) ). Note, however, that they applied $1^{\circ} \times 1^{\circ}$ boxcar smoothing to reduce the pixel-to-pixel noise level, which could blur image resolution in many cases. The recent space observation with the Solar Mass Ejection Imager (SMEI) provided nearly all-sky photometric maps for over 5 years with a moderate spatial resolution of 30' Buffington et al. 2009). Note that Buffington et al. (2009) stacked data over the mission period because the $\mathrm{S} / \mathrm{N}$ ratio was inadequate to produce the Gegenschein map. They did not consider the seasonal variation in the Gegenschein morphology.

The first question we would like to address in this paper is the exact position of the maximum brightness in the Gegenschein. Dumont (1965) and Roosen (1970a, b ) found that the maximum brightness was close to the antisolar point. However, Tanabe (1965) and Wolstencroft \& Rose (1967) reported that the maximum brightness of the Gegenschein was displaced from the antisolar point and existed close to the invariable plane of the Solar System. Mukai et al. (2003) argued that the maximum brightness was largely influenced by the existence of fine-scale structures in the zodiacal cloud, i.e., asteroidal dust bands (Sykes \& Greenberg 1986; Sykes 1990; Reach et al. 1997), based on their CCD observation. After subtraction of the fine structures, they estimated a deviation from the antisolar point of $-0.4^{\circ}$ from an image taken on 2 November 1997. Buffington et al. (2009) found that the maximum existed at the antisolar point, although the study was based on SMEI measurements, whose spatial resolution $\left(0.5^{\circ}\right)$ is equivalent to the displacement discussed in Mukai et al. (2003) and Ishiguro et al. (1998). High-resolution observations $\left(<0.5^{\circ}\right)$ are thus essential to settle the matter of the maximum brightness position.

The second question concerns the Gegenschein morphology. A systematic morphological change was pointed out by Tanabe (1965) and later confirmed by high-resolution observations (James et al. 1997; Ishiguro et al. 1998; Kwon et al. 2004). This change can be caused by the discrepancy between the orbital plane of the Earth (i.e., the ecliptic plane) and the plane of symmetry of the zodiacal cloud. The spatial distribution of the zodiacal cloud has been investigated around the solar elongation $\epsilon \sim 90^{\circ}$ (Kelsall et al. 1998). However, little is known about the spatial distribution around the Gegenschein region, which might retain information about the spatial distribution of interplanetary dust particles that exist further than those observed around $\epsilon \sim 90^{\circ}$.

In this paper, we present high-resolution images of the Gegenschein taken by a wide-field CCD camera for the diffuse night sky, Wide-field Imager of Zodiacal light with ARray Detector (WIZARD). Both the sensitivity and the spatial resolution are better than those of the instruments used previously (James et al. 1997; Ishiguro et al. 1998; Mukai et al. 2003; Buffington et al. 2009; Kwon et al. 2004). We examined the morphology and the photometric center of the Gegenschein for 4 years using WIZARD. We succeeded in detecting the narrow brightness enhancement at the exact 
position of the antisolar direction. The maximum brightness of the Gegenschein consistently existed at the antisolar point throughout our observation period. We examined the volume scattering phase function around the opposition and considered the reason for the narrow brightness enhancement. In Section 2, we describe the observations and the data reduction. In Section 3, we show the morphological maps of the Gegenschein. In addition, we discuss the annual variation in the peak position. In Section 4, we test several phenomenological three-dimensional models to interpret the observational results. Finally, we discuss the geometric albedo of interplanetary dust particles in the last section.

\section{Observations}

\subsection{The Instrument and its Performance}

All data in this paper were taken with WIZARD. A detailed description of the instrument is given in Usui et al. (2002), Ishiguro et al. (2003), and Ueno et al. (2007). In this section, we briefly describe the instrument and report on its performance based on the data reduction results.

WIZARD was developed to cover a wide-extended zodiacal light and the Gegenschein. It consists of a camera head and a wide-field lens (Figure 1). The system was mounted on an equatorial mounting. We employed a back-illuminated full-frame CCD chip, EEV CCD 42-80 (27.6 × 55.3 mm; 2048×4096 pixels), placed in a compact $\mathrm{N}_{2}$-cooled cryogenic dewar (Kadel Engineering Corp.). During observations, the CCD temperature was maintained at around $180 \mathrm{~K}$. The optical system was designed by the Genesia Corporation. It has a large image circle (60 $\mathrm{mm}$ in diameter) to cover the whole imaging area of the CCD. The focal ratio and the focal length are 2.8 and $32.5 \mathrm{~mm}$, respectively. With the camera head, it gives a field of view of $46^{\circ} \times 92^{\circ}$ with a pixel resolution of $1.4^{\prime}$. Figure 2 shows the transmittance of the optical filter used for zodiacal light observations. It covers a wavelength range of 440-520 nm, avoiding prominent airglow emissions and artificial emission light. In particular, it was designed not to transmit O I airglow at $557.7 \mathrm{~nm}$ and artificial mercury vapor light at $435.8 \mathrm{~nm}$. In addition, sodium beacons at $589.2 \mathrm{~nm}$ as laser guide stars are nowadays problematic for astronomical observations. The transmittances at these wavelengths are negligible, that is, $0.35 \%$ at $435.8 \mathrm{~nm}(\mathrm{Hg}), 0.19 \%$ at $557.7 \mathrm{~nm} \mathrm{O} \mathrm{I}$, and $0.0008 \%$ at $589.2 \mathrm{~nm}(\mathrm{Na})$, respectively. The CCD clock pattern and the operation commands were prepared on a Linux host computer and sent to the CCD chip through a COGITO-3 system. COGITO-3 is a programmable control system for imaging devices specifically designed for scientific purposes and was developed by Ueno and Wada at ISAS/JAXA. It contains three units: a sequencer board, an A/D board, and a driver board. The obtained analog signal was converted to the digital one on the COGITO-3 A/D board and transferred to the host computer via a PCI-VME bus interface adapter.

The analog signal in each pixel was sampled 16 times to reduce the readout noise. In addition, during CCD readout, we turned off the pulse motor used for star-tracking to reduce noise associated with the motor drive unit. Consequently, the readout noise was reduced to the level of 20-25 $\mathrm{e}^{-}$. 
To monitor the zero level of the CCD, we recorded the overscan data by reading 50 dummy pixels before and after each row was read. For confirmation, we examined the stability of the zero intensity level using dark frames obtained at the intervals of the light frame. We found that the standard deviation of the zero level in each frame was $\lesssim 10 \mathrm{e}^{-}$. Using the overscan region, we determined the zero level to an accuracy of $4 \mathrm{e}^{-}$. The typical brightness of the Gegenschein plus airglow was about $1.1 \mathrm{e}^{-} \mathrm{s}^{-1}$. With an exposure time of $600 \mathrm{~s}$, the uncertainty of the zero intensity level (i.e., $\left.4 \mathrm{e}^{-}\right)$was small enough ( $0.6 \%$ of the Gegenschein plus airglow brightness, i.e., $\left.660 \mathrm{e}^{-}\right)$to derive the brightness of the Gegenschein plus airglow. In addition, background noise becomes dominated by photon noise from the natural night sky background rather than the readout noise. For this reason, we fixed the exposure time of $600 \mathrm{~s}$ for our Gegenschein observations.

An image taken by a wide-field camera generally shows strong vignetting. The detected intensity around the optical center is thus brighter than that at the edge of the frame. A flat-field correction is essential to obtain accurate intensity maps of the zodiacal light and the Gegenschein. It is, however, difficult to obtain flat data using WIZARD because of its huge field of view. We investigated flat-fielding for different spatial scales, that is, by considering optical vignetting as a low-frequency component and pixel-to-pixel variation in the sensitivity as a high-frequency component. We examined the optical vignetting by observing bright stars at different positions on the CCD chip. We confirmed that the weather conditions were stable and the airmasses of these stars were constant during the exposures, suggesting that the brightnesses of these light sources were stable enough to examine the vignetting. Figure 3 shows the vignetting function across the CCD chip. From the measurement, we found that the detected intensity was almost constant within $20^{\circ}$ from the optical center and reduced by $15 \%$ at $38^{\circ}$ and $30 \%$ at $47^{\circ}$. For reference, we compare

the vignetting function with those of previous CCD observations of the Gegenschein (James et al. 1997; Ishiguro et al. 1999a), in which commercial products for single-lens reflex cameras were used. It is clear that the optics for WIZARD is well designed to suppress the vignetting throughout a large portion of the CCD chip. Small-scale (pixel-to-pixel) fluctuations of the CCD sensitivity were examined by taking images with a Labsphere integrating sphere. We found that the pixel-to-pixel responsivity variation was negligibly small $(\lesssim 0.3 \%)$.

The performance of WIZARD, which are critical for estimating the error in the measurements, are summarized in Table 1.

\subsection{Observations and Data Analysis}

In normal operation, we began observation soon after the end of evening twilight. We took a couple of images successively without slewing the camera to its next position. Since the motor controller for star-tracking was turned off for about 3 min after exposures for the CCD readout, the position of celestial objects moved about one degree on the CCD chip. This observation strategy enables us to distinguish small-scale structures of the Gegenschein from instrumental artifacts associated with uneven sensitivity of the instrument. 
We made a sequence of observations of the zodiacal light and the Gegenschein with WIZARD between March 2003 and November 2006 on Mauna Kea. Our observations were supported by the Subaru telescope (National Astronomical Observatory of Japan) and the InfraRed Telescope Facility (ITRF; NASA and Institute for Astronomy, University of Hawaii). We observed zodiacal light and the Gegenschein for 45 nights under photometric conditions. We selected Gegenschein data taken at high Galactic latitude near the zenith. In total, 14 nights of data in different seasons of the year were analyzed for the present study. We summarize the observation conditions of these data in Table 2. Gegenschein data were not available in May-July and December-January because the antisolar point was close to the Galactic plane (Figure 4).

The night sky brightness observed from the ground consists of those of zodiacal light including the Gegenschein, integrated starlight of unresolved stars, diffuse Galactic light, and pseudocontinuum airglow (Leinert et al. 1998). These light sources are attenuated by scattering of atmospheric molecules and aerosols. At the same time, the scattered component in Earth's atmosphere results in a diffuse light source. Dumont (1965) introduced the concept of effective optical depth for diffuse light sources, which is different from that for point sources. Later, Hong et al. (1998) and Hong et al. (2002) developed the idea by solving radiative transfer in an anisotropically scattering spherical atmosphere of the Earth. It is difficult reduce zodiacal light data in high-airmass (i.e., large zenith distance) regions owing to the considerable contributions of the atmospheric diffuse light and the strong zenith extinction, although some intensive studies were conducted to obtain the absolute brightness of the zodiacal light from ground-based observatories (Kwon et al. 2004; Dumont 1965). The data reduction of the Gegenschein is, however, relatively easy. The Gegenschein appears near the zenith around midnight on Mauna Kea, where the contaminations from airglow and zenith extinction are smallest in the sky. The typical brightness of atmospheric diffuse light components was $20 \mathrm{~S}_{\odot 10}$ and had a weak slope of $10 \%-20 \%$ of the Gegenschein brightness over a $40^{\circ}$ field of view in Gegenschein exposures, where $1 \mathrm{~S}_{\odot 10}$ is equivalent to the flux of a solartype star of magnitude 10 distributed over one degree squared (Leinert et al. 1998). Inadequate reduction for these atmospheric components does not tend to lead to a misunderstanding of the morphology of the Gegenschein. In addition to these diffuse light sources mentioned above, there remain a number of point sources. Stars brighter than a visual magnitude of 12-14 (depending on the Galactic latitude) were resolved by our observations. We subtracted point sources brighter than magnitude 12 using the USNO-B2.0 catalog (Monet et al. 2003). The detected pixel data were replaced by the median values of circumjacent pixels.

For the most part, the data were analyzed as follows: First, the raw data were reduced using the overscan region as a bias level plus flat-field data. The flux calibration factor and the optical depth of the point sources are determined by aperture photometry of field stars at various airmasses. Since we applied a nonstandard filter, we constructed a photometric standard star catalog for WIZARD data. An empirical formula is applied to convert from the Johnson-Cousins system into the WIZARD system, that is, $M_{W Z D}=V+\alpha(B-V)$, where $\alpha$ is the color conversion factor and $B$ and $V$ are B-band and V-band magnitudes, respectively. To derive the coefficient $\alpha$, we selected 56 stars in 
BSC Ver. 5 in an airmass range of 1.00 to 1.07. Since the typical extinction coefficient on Mauna Kea in the WIZARD band was $\sim 0.15$, atmospheric extinction did not change the stellar magnitude by $\gtrsim 0.01$ magnitude in this sky area, which is adequate to derive the coefficient $\alpha$. The result is shown in Figure 5. In the figure, the bottom axis denotes the instrumental magnitude measured by aperture photometry, and the perpendicular axis denotes the magnitude in the WIZARD system $\left(M_{W Z D}\right)$. The best-fit parameter was found to be $\alpha=0.605 \pm 0.005$.

Unresolved starlight brightness plus diffuse Galactic light brightness were subtracted by using Pioneer 10 and 11 data, which was obtained beyond the inner Solar System (i.e., outside the zodiacal cloud) and the USNO-B2.0 catalog (Monet et al. 2003). Although the central wavelength of WIZARD $(460 \mathrm{~nm})$ is slightly longer than that of the Pioneer blue band (440 nm), we used the Pioneer data without color correction. The contribution from unresolved starlight (stars fainter than magnitude 12) was estimated to be $20 \mathrm{~S}_{\odot 10}$ (or $10 \%$ of the Gegenschein brightness) at a Galactic latitude of $25^{\circ}$ and $10 \mathrm{~S}_{\odot 10}$ (or $5 \%$ of the Gegenschein brightness) at a Galactic latitude of $50^{\circ}$ (Leinert et al. 1998). The intensity of the airglow was subtracted using Barbier's function (Barbier 1944; Barbier et al. 1961), whereas the intensity of the multiplescattering component in Earth's troposphere was not subtracted because of its weak contribution in our data around the Gegenschein. The extinction coefficients of Earth's atmosphere were obtained through stellar photometry, while the effective optical depths for the extended sources were obtained using the QDM_sca code by adjusting the particle albedo $\omega$ and the asymmetry factor $g$ (Hong et al. 1998, 2002) to match the brightness of previous zodiacal light observations (Levasseur-Regourd \& Dumont 1980). Some of unknown parameters such as the intensity of the airglow at zenith and scaling factor of starlight brightness by Pioneer to our system were determined empirically to fit our data to the zodiacal light brightness in Levasseur-Regourd \& Dumont (1980). We derived the Gegenschein brightness in the range of 165-190 $\mathrm{S}_{\odot 10}$. Throughout this data reduction, we found that there is an uncertainty of $\pm 10-15 \mathrm{~S}_{\odot 10}$ in the absolute brightness. This uncertainty is mainly caused by inadequate subtraction of atmospheric diffuse light sources and imperfect correction of the zenith extinction, although instrumental effects such as scattered light inside the camera cannot be ruled out. It is, however, emphasized that the relative intensity

distribution of $\lesssim 20^{\circ}$ structures is reliable in our data set because it cannot be influenced by the inevitable atmospheric and instrumental effects.

\section{Results}

\subsection{Observational Results}

Figure 6 6 shows snapshots of the Gegenschein in ecliptic coordinates. The long axis was nearly parallel to the ecliptic and stretched in the east-west direction. The morphology was symmetrical with respect to the line at an ecliptic longitude with respect to the Sun of $\lambda-\lambda_{\odot}=180^{\circ}$, where $\lambda$ and $\lambda_{\odot}$ denote the ecliptic longitude and ecliptic longitude of the Sun. Looking at the morphologies 
closely, we see a truncated oval shape whose eastern and western edges are angular rather than round. As the antisolar point is approached, the shape changes from truncated oval to round. There is a prominent peak at the antisolar point.

It is clear that the brightness distribution shifted northward on 17 February and southward on 12 October (Figure [6). We found that the Gegenschein morphology deviated to the south in August-October and to the north in February-April. This trend is consistent with the result of Ishiguro et al. (1998), who mentioned that the overall brightness distribution shifted southward in September but shifted northward in March. The annual variation can be explained by the threedimensional zodiacal cloud model having a plane of symmetry deviated southward during August and November and northward during February and April, as previously noticed in Ishiguro et al. (1998). We discuss the annual variation in Section 3.2.

Figure 7 shows the surface brightness profile of the Gegenschein on 12 October at three different longitudes. These profiles show a convex feature over the ecliptic latitude range $\beta=-3^{\circ}$ to $1^{\circ}$. The flat-topped profiles were detected for the first time through the infrared observation of zodiacal emission (Low et al. 1984). They were also recorded for the Gegenschein (Ishiguro et al. 1999a; Mukai et al. 2003). Recent theoretical studies have showed that features in the profiles are associated with materials generated by recent catastrophic collisions in the main-belt asteroid region (Nesvorný et al. 2003, 2006). However, the convex structure is not clear in the profile of $\lambda-\lambda_{\odot}=180^{\circ}$. Instead, it exhibits a steep increase at $\beta=0^{\circ}$.

Despite the annual variation in the overall shape, we found that the maximum position coincided with the antisolar point through the observation runs. Neither east-west nor north-south displacement was detected in the profiles at $\lambda-\lambda_{\odot}=180^{\circ}$. Figure 8 shows the ecliptic latitude of the maximum intensity at $\lambda-\lambda_{\odot}=180^{\circ}$. For comparison, we show photoelectric and photographic data compiled by Roosen $(1970 \mathrm{~b})$ as well as previous CCD data. Our results are inconsistent with those in Roosen (1970b), James et al. (1997), Ishiguro et al. (1998), and Mukai et al. (2003), but

they are consistent with the recent measurement from space by SMEI (Buffington et al. 2009). It should be emphasized that our measurement is the most reliable of all observational data so far because of the better spatial resolution.

\subsection{Comparison with 3-D Empirical Dust Distribution Models}

In this chapter, we analyze the annual variation in the morphology and the peak position by conducting three-dimensional zodiacal cloud model simulations.

The surface brightness of the zodiacal light as seen by an observer in the ecliptic plane at an ecliptic latitude $\beta$ and an ecliptic longitude $\lambda-\lambda_{\odot}$ with respect to the Sun can be described as a double integral over the size distribution and along the line of sight. As an approximation, we assume the following: 
1. The size distribution of dust particles in the zodiacal cloud is independent of their position in interplanetary space.

2. The albedo and scattering phase functions of dust particles are independent of position.

3. The zodiacal cloud has a plane of symmetry and is axisymmetric with respect to an axis perpendicular to the plane of symmetry.

4. Dust grains are sufficiently far from the Sun that we can regard the Sun as a point source.

Under these assumptions, the brightness of the zodiacal light is given by

$$
I_{Z L}\left(\lambda-\lambda_{\odot}, \beta\right)=\int_{0}^{\infty} \int_{0}^{\infty} F\left(r_{h}\right) n(x, y, z) f(s) \sigma_{s c a}(s) \phi(\theta ; s) d s d l
$$

where $d l$ and $\theta$ denote the line element and the scattering angle, respectively, and $s$ is the particle radius. $f(s)$ is the differential size distribution of the particles with a radius of $s . \sigma_{s c a}(s)$ is the cross section of the particles. $\phi(\theta ; s)$ characterizes the distribution of scattered intensity with $\theta$. The incident solar flux $F\left(r_{h}\right)$ can be replaced by $F_{\odot}\left(r_{0} / r_{h}\right)^{2}$, where $F_{\odot}$ denotes the solar flux at the heliocentric distance $r_{h}=r_{0} . n(x, y, z)$ is the number density of the cloud particles in ecliptic coordinates with respect to the Sun, where the $x$ axis points from the Sun toward the vernal equinox, the $z$ axis is perpendicular to the ecliptic plane, and the $y$ axis completes a right-handed orthogonal coordinate system. The geometry and the notations are summarized in Figure 9 .

For simplification, the mean volume scattering phase function $\Phi(\theta)$ and the mean total scattering cross section $\bar{\sigma}_{\text {scat }}$ are defined by

$$
\int_{0}^{\infty} f(s) \sigma_{\text {scat }}(s) \phi(\theta ; s) d s \equiv \bar{\sigma}_{\text {scat }} \Phi(\theta)
$$

with a normalization condition, that is,

$$
\int_{4 \pi} \Phi(\theta) d \Omega=1
$$

The mean volume scattering phase function was empirically derived from the zodiacal light observations (Leinert et al. 1976; Hong 1985); Lamy \& Perrin 1986). Figure 10 (left) shows an example of the mean volume scattering phase function given by Hong (1985) and Lamy \& Perrin (1986). It has a strong peak in the forward direction, an isotropic component at intermediate scattering angle, and a slight enhancement in the backward direction.

The spatial distribution of dust particles has been discussed (Giese et al. 1986; Giese \& Kneissel 1989; Kelsall et al. 1998). In the early years of research, the spatial distribution was of a form separable into radial and vertical terms, that is,

$$
n\left(r_{h} ; \beta^{\prime}\right)=n_{0}\left(\frac{r_{h}}{r_{0}}\right)^{-\nu} h\left(\beta^{\prime}\right)
$$


where $n_{0}$ is the reference dust number density on the symmetry plane at heliocentric distance $r_{h}=r_{0}$. The radial power law is motivated by the radial distribution expected for particles under the effect of Poynting-Robertson drag, which results in $\nu=1$ for dust particles in circular orbit; $h\left(\beta^{\prime}\right)$ describes how the dust number density reduces as we move away from the symmetry plane. Various axisymmetric distribution models were proposed. Among them, the ellipsoid model,

$$
h\left(\beta^{\prime}\right)=\left[\left(1+6.5 \sin \beta^{\prime}\right)^{2}\right]^{-0.65},
$$

and the fan model,

$$
h\left(\beta^{\prime}\right)=\exp \left(-2.1\left|\sin \beta^{\prime}\right|\right),
$$

were often applied in phenomenological model calculation in the literature (e.g., Giese \& Kneissel 1989). The plane of symmetry was derived by different authors (see, e.g., Kwon et al. (2004), who obtained $i_{\text {sym }} \sim 2^{\circ}$ and $\Omega_{\text {sym }}=80^{\circ}$, and Mukai et al. (2003), who obtained $i_{\text {sym }}=2.03^{\circ} \pm 0.50^{\circ}$ and $\Omega_{\text {sym }}=54^{\circ}-64^{\circ}$ ). A sophisticated model, in which localized dust condensations as well as the axisymmetric smooth component are considered, was suggested on the basis of infrared observations with the Cosmic Background Explorer's Diffuse Infrared Background Experiment (COBE/DIRBE) (Kelsall et al. 1998). It consists of a smooth diffuse component that has a plane of symmetry of $i_{\text {sym }}=2.03^{\circ} \pm 0.017^{\circ}$ and $\Omega_{\text {sym }}=77.7^{\circ} \pm 0.6^{\circ}$, three asteroidal dust components, and circumsolar ring components.

We began with a comparison of the ellipsoid model and the fan model. We assumed a plane of symmetry of $i_{\text {sym }}=2^{\circ}$ and $\Omega_{\text {sym }}=80^{\circ}$ based on previous studies (Kwon et al. 2004; Kelsall et al. 1998). The radial power law exponent $\nu=1.3$ is assumed. We confirmed that $\nu$ does not change the Gegenschein morphology significantly within $\sim 30^{\circ}$ from the antisolar point. The mean volume scattering phase function in Hong (1985) was applied. We should note here that the power-law exponent $\nu$ is insensitive to the morphology of the Gegenschein, as already pointed out in Hong (1985). For the reason, we did not give much attention to $\nu$ in this paper. The results are shown in Figure 11. The systematic change in north-south asymmetry is explained qualitatively by these models. The model morphologies are, however, different from the observed ones in that they have protruding edges toward the eastern and western directions (see the arrows in Figure 11). No peak in the antisolar direction appears in these modeled images. These mismatches cannot be explained by different values of $i_{\text {sym }}$ and $\Omega_{\text {sym }}$, suggesting that the classical models are inadequate for explaining the observed distribution of interplanetary dust particles.

Next, we applied the infrared model of Kelsall et al. (1998). Because the model was constructed to fit the data in near- and far-infrared wavelengths, no scattering phase function was provided for optical wavelengths. We again applied the scattering phase function in Hong (1985). The upper panels in Figure 12 show the results using only the smooth component of the infrared model, while the middle panels show the results using all the components. From the comparison between top and middle panels, it is clear that the angular shape is associated with fine-scale structure, in particular, the dust-band components. Both the north-south asymmetry and the angular edge along the eastwest direction were reproduced by the model (Figures [12(c) and (d)). It is interesting to note that 
the model was constructed based on infrared observations and yet fit the optical zodiacal light as well. In addition, the infrared observations covered the zodiacal emission at solar elongation of $64^{\circ}-$ $124^{\circ}$. Both the observed wavelengths and the solar elongation angle of the infrared observations differ from those of our observations. Nevertheless, the infrared model matches the Gegenschein in the visible wavelength range.

In Figures 12 and 6, we would like to draw attention to the antisolar point, where the brightness spike is clearly seen in our observational images but not visible in the model image. The difference is also seen in Figure 13, where the observed brightness profile around the antisolar point does not match the observed one. This is probably because the backscattering part of the scattering phase function is inadequate for reproducing the observed spikes. Since the scattering phase function has so far been derived using low-resolution data, it is likely that the backscattering part within $\sim 1^{\circ}$ has not been considered yet. For this reason, we modified the scattering phase function by multiplying it by a term used in Kaasalainen et al. (2003),

$$
\Phi^{\prime}(\theta)=\left\{1+A \exp \left(-\frac{\pi-\theta}{B}\right)\right\} \Phi(\theta)
$$

where $A$ and $B$ are fitting parameters and $\Phi(\theta)$ is the original scattering phase function in Hong (1985). We derived $A=0.15$ and $B=0.021$ (or $1.2^{\circ}$ ) to match the observed images. With the modified scattering phase function, we fit the surface brightness profiles (see Figure 12 (e)-(f) indicated by arrows, and also Figure 13).

\section{Discussion}

The question we have to address here is whether the brightest spot on the Gegenschein was caused by scattered light from interplanetary dust particles, by an instrumental effect (i.e., uneven sensitivity of CCD pixels or vignetting), or by other light sources such as stars and galaxies. Since we detected the brightness enhancement in different positions on the CCD, it is unlikely that the brightness enhancement was caused by instrumental effects. In addition, because the antisolar position moves relative to stars and galaxies at a rate of $1^{\circ}$ day, we can distinguish zodiacal light components from stellar objects by comparison with images taken on different nights. For these reasons, it is clear that the brightness enhancement originates from the interplanetary dust cloud, because it always appears at the antisolar point.

Our observational result indicates that the volume scattering phase function sharply increases close to a phase angle of zero (that is, phase angle $=180^{\circ}$ - scattering angle $\theta=0^{\circ}$ ). The sharp increase (hereafter referred to as the opposition spike) is a common phenomenon inherent to airless bodies in the Solar System, such as the Moon, asteroids, satellites of the major planets, Saturn's rings (Mishchenko 1993), and Kuiper Belt objects (Sheppard and Jewitt 2002); it is characterized by a nonlinear increase in surface brightness as the phase angle decreases to zero. Through this effect, the amplitude and the width of the scattered light change significantly within $1^{\circ}-5^{\circ}$. There are 
two main physical mechanisms to explain the opposition effect on a surface covered with regolith: shadow-hiding and coherent backscattering enhancement. Shadows directly opposite the Sun are hidden by the scatterers, and the absence of visible shadow makes the area brighter on average (the shadow-hiding effect). On the other hand, coherent backscattering is an effect that describes the appearance of an intensity cone when photons traveling in a time-reversed path self-interfere constructively in the backscattered direction.

The opposition effect has been detected to occur on airless bodies in the Solar System. Shadowhiding and coherent backscattering enhancement have been proposed as reasons for the opposition effect observed in regolith layers (Muinonen et al. 2002). It is interesting to note that the opposition effect was detected in the interplanetary dust cloud from our observations. This evidence may suggest that scattering by a single interplanetary dust particle induces an opposition surge. Shkuratov \& Helfenstein (2001) predicted from their model, in which the aggregate structure of regolith is considered, that a single regolith particle could exhibit its own opposition effect. Hapke (2002) also emphasized that multiple scatterings can occur between different parts of a single particle. Min et al. (2010) showed that the large $(>100 \mu \mathrm{m})$ dust grains covered by regolith particles are responsible to the strong backward scattering observed in the Fomalhaut debris disk (Le Bouquin and al. 2009). Further researches involving multiband observations, polarimetric observation and theoretical studies are required to study the mechanisms responsible for the opposition effect observed in the Gegenschein. Although we cannot conclude whether the opposition effect in the Gegenschein is caused by the shadow-hiding effect or interference or both, we can safely conclude that the effect is induced by scatterers whose sizes are significantly larger than the optical wavelength.

The albedo of interplanetary dust particles leads us to consider their origin. This has been investigated in literature; Cook (1978) studied the albedo using micrometeoroid experiment data onboard Helios A and Pioneer 10 as well as zodiacal light observational data and derived an albedo ranging from 0.07 at $0.1 \mathrm{AU}$ from the Sun through 0.006 at $1 \mathrm{AU}$ and down to 0.0001 at 3.3 AU. Later, Hanner (1980) reanalyzed the data to obtain a mean geometric albedo of 0.24 for dust particles near 1 AU. Dumont \& Levasseur-Regourd (1988) and Renard et al. (1995) studied zodiacal light and zodiacal emission by using the node of lesser uncertainty method, and they obtained an albedo at $1 \mathrm{AU}$ of $0.08-0.15$ at a scattering phase function of $90^{\circ}$, which translates into a geometric albedo of 0.16-0.30 with the scattering phase function by Hong (1985). Lumme \& Bowell (1985) derived an albedo of 0.04 using an empirical polarimetric method. All these measurements are inconsistent with each other and have not yet led to a discussion about the origin.

Our observational data can be used to derive an accurate albedo value in combination with the infrared model. Because our observed quantity is obtained at the opposition direction from the Sun, it gives straightforward input for deriving the geometric albedo of interplanetary dust particles around the Earth orbit (Appendix A.) because it is defined at zero phase angle. In addition, the good consistency between the optical and infrared spatial distributions of dust particles suggests that the effective size of dust grains in the optical wavelength region is almost identical to that at infrared wavelengths. This result supports the idea that large grains $(>10 \mu \mathrm{m})$ are responsible for 
the scattering cross section of zodiacal light (Giese \& Gruen 1976).

In light of this large-particle hypothesis, let us derive the geometric albedo. We rewrite Eq. (1) at the antisolar point using the geometric albedo $A_{p}\left(180^{\circ}\right)$ (Hanner et al. 1981) as follows:

$$
I_{Z L}\left(180^{\circ}, 0^{\circ}\right)=\int_{0}^{\infty} F\left(r_{h}\right) n(x, y, z) \frac{A_{p}\left(180^{\circ}\right)}{\pi} d l .
$$

Because $n(x, y, z)$ is given by the DIRBE infrared model while the intensity of zodiacal light at opposition is obtained by our observation $\left(175 \pm 15 \mathrm{~S}_{\odot 10}\right)$, we can deduce a mean geometric albedo of $A_{p}\left(180^{\circ}\right)=0.07 \pm 0.01$. The DIRBE infrared model also suggests that about $7 \%$ of the geometric cross-sectional area accounts for asteroidal particles, particularly the inner dust band from the Karin collisional family (whose mean geometric albedo is $0.20-0.22$; Harris et al. (2009); Usui et al. (2011)) and the circumsolar resonance ring possibly originating from the asteroidal belts. Given that $7 \%$ of the geometric cross section is accounted for by particles with $A_{p}\left(180^{\circ}\right)=0.20-0.22$, we found that the remaining zodiacal light (the smooth component in the DIRBE model) should have a geometric albedo of $0.06 \pm 0.01$.

Our result is consistent with the fact that the micrometeorites accumulated around Earth orbit have low albedo $(<0.1$; Brownlee (1978); Hanner (1980)). Among Solar System minor objects, comet nuclei and C- and D-type asteroids show low albedo, that is, 0.02-0.06 for comet nuclei (Lamy et al. 2004) and $0.07 \pm 0.04$ for C-type asteroids (Usui et al. 2011). Recent dynamical simulations of interplanetary dust particles suggest that $85 \%-95 \%$ of the observed mid-infrared emission is produced by particles from Jupiter-family comets and $<10 \%$ by dust from long-period comets (Nesvorný et al. 2010). Jewitt et al. (2013) conclude that $<3 \%$ of the interplanetary dust particles are produced by events like P/2010 A2 probably caused by breakup of an asteroid (Snodgrass et al. 2010; Jewitt et al. 2011). On the contrary, Tsumura et al. (2010) insist that high-albedo particles dominate near Earth orbit based on their spectroscopic observation at near-infrared wavelengths. Although further investigation is required on the fraction of asteroidal particles with respect to cometary particles, it seems plausible that majority of interplanetary dust particles have a composition similar to carbonaceous chondrites rather than siliceous particles.

\section{Summary}

In this paper, we outlined the instrumentation, the data reduction, and the observations of the Gegenschein using the hand-made observation system WIZARD. Our main findings are as follows:

- A narrow brightness enhancement was superimposed on the smooth gradient of the Gegenschein at the antisolar point. Hence, the position of maximum brightness exists at the antisolar point throughout the year.

- The Gegenschein morphology changed according to the orbital motion of the Earth. It is well produced by a model constructed using infrared observations by COBE/DIRBE. 
We hypothesized that the zero phase angle spike is caused by coherent backscattering and/or shadow-hiding effects on the rough surfaces of individual dust particles. The above results indicate that the effective sizes of interplanetary dust particles responsible for optical zodiacal light and infrared zodiacal emission are much larger than those corresponding to the observed wavelengths.

Finally, we derived the geometric albedo of interplanetary dust particles using our observational result and found that it is $0.06 \pm 0.01$ for the smooth component of the zodiacal cloud. The derived albedo is in accordance with that of the dark micrometeorites accumulated around earth orbit and the observed cometary dust particles. We concluded that chondritic particles are dominant near Earth space.

We would like to express out greatest gratitude to the people who have helped and supported us throughout our project. In particular, we are grateful to Prof. Seung Soo Hong for his continuous encouragement and advices from conceptual inception of the project to these days. We also wish to thank stuffs at IRTF and Subaru (NAOJ), especially, Alan Tokunaga, George Koenig, Kazu Sekiguchi, Masao Nakagiri, and Akihiko Miyashita, for their tremendous contribution and support towards the long-term observations at Mauna Kea. The laboratory experiments were made at University of Tokyo. The control software of COGITO was developed by Takehiko Wada (ISAS), Yosuke Ohno (RIKEN) and Hitoshi Muller (Musashino Art University), and modified by Ryosuke Nakamura (NICT). The optical system was designed and fabricated by Genesia Corporation, Japan. Shoko Ohtsuki (Senshu University), Yuki Sarugaku (ISAS), Naoya Miura, Hideo Sagawa, Seitaro Urakawa helped us. The performance test at a laboratory was supported by a grant of Seoul

National University. MI and HY are supported by National Research Foundation of Korea (NRF) grant funded by the Korea Government (MEST) (No. 2012R1A4A1028713).

\section{A. Appendix A: Line of Sight Depth of Zodiacal Light and Zodiacal Emission}

Because the intensities of optical zodiacal light and infrared zodiacal emission are quantities integrated along the line of sight, it is difficult to specify the particles we observe. We consider the contribution of the elements at different distance from the observers to the total flux of optical zodiacal light and infrared zodiacal emission. Figure 14 show the contribution in different wavelengths at $\lambda-\lambda_{\odot}=90^{\circ}$ and $\lambda-\lambda_{\odot}=180^{\circ}$ on the ecliptic plane. We found that the line of sight depth becomes shallowest at $12 \mu \mathrm{m}$ because hot dust particles $(\sim 280 \mathrm{~K})$ near Earth orbit are efficient infrared emitters. On the other hand, distant particles contribute well at longer wavelength. The depth in the optical wavelength is similar to that at $25 \mu \mathrm{m}$. It seems that we derived the albedo of interplanetary dust particle within $\sim 2-3$ AU from the Earth. 


\section{REFERENCES}

Barbier, D. 1944, Ann. Geophys., 1, 144.

Barbier, D., Dufay, J., \& Williams, D. 1961, Ann. Astrophys., 14, 399

Brownlee, D. E. 1978, Cosmic Dust, 295

Buffington, A., Bisi, M. M., Clover, J. M., Hick, P. P., Jackson, B. V., Kuchar, T. A., \& Price, S. D. 2009, Icarus, 203, 124

Cook, A. F. 1978, Icarus, 33, 349

Dumont, R. 1965, Ann. Astrophys., 28, 265

Dumont, R. \& Sanchez, F. 1975, A\&A, 38, 397

Dumont, R. \& Levasseur-Regourd, A.-C. 1988, A\&A, 191, 154

Giese, R. H. \& Gruen, E. 1976, Interplanet. Dust Zodiacal Light, 48, 135

Giese, R. H., Kneissel, B., \& Rittich, U. 1986, Icarus, 68, 395

Giese, R. H. \& Kneissel, B. 1989, Icarus, 82, 369

Hanner, M. S. 1974, BAAS, 6, 338

Hanner, M. S. 1980, Icarus, 43, 373

Hanner, M. S., Giese, R. H., Weiss, K., \& Zerull, R. 1981, A\&A, 104, 42

Hong, S. S. 1985, A\&A, 146, 67

Hong, S. S., Kwon, S. M., Park, Y.-S., \& Park, C. 1998, Earth, Planets Space, 50, 487

Hong, S. S., Park, Y.-S., Kwon, S. M., Park, C., \& Weinberg, J. L. 2002, J. Korean Astron. Soc., 35,41

Harris, A. W., Mueller, M., Lisse, C. M., \& Cheng, A. F. 2009, Icarus, 199, 86

Ishiguro, M., Fukushima, H., Kinoshita, D., Mukai, T., Nakamura, R., Watanabe, J.-I., Watanabe, T., \& James, J. F. 1998, Earth, Planets Space, 50, 477

Ishiguro, M., Nakamura, R., Fujii, Y., Morishige, K., Yano, H., Yasuda, H., Yokogawa, S., \& Mukai, T. 1999, ApJ, 511, 432

Ishiguro, M., Nakamura, R., Fujii, Y., \& Mukai, T. 1999, PASJ, 51, 363

Ishiguro, M., Mukai, T., Nakamura, R., Usui, F., \& Ueno, M. 2002, IAU Colloq. 181: Dust in the Solar System and Other Planetary Systems, 98 
Ishiguro, M., et al. 2003, Proc. SPIE, 4842, 366

James, J. F., Mukai, T., Watanabe, T., Ishiguro, M., \& Nakamura, R. 1997, MNRAS, 288, 1022

Jewitt, D., Stuart, J. S., \& Li, J. 2011, AJ, 142, 28

Jewitt, D., Ishiguro, M., \& Agarwal, J. 2013, ApJ, 764, L5

Kaasalainen, S., Piironen, J., Kaasalainen, M., et al. 2003, Icarus, 161, 34

Kelsall, T., et al. 1998, ApJ, 508, 44

Kwon, S. M., Hong, S. S., \& Weinberg, J. L. 2004, New Astron., 10, 91

Lamy, P. L., \& Perrin, J.-M. 1986, A\&A, 163, 269

Lamy, P. L., Toth, I., Fernandez, Y. R., \& Weaver, H. A. 2004, Comets II, 223

Le Bouquin, J.-B., Absil, O., Benisty, M., et al. 2009 aap, 498, L41

Leinert, C., Link, H., Pitz, E., \& Giese, R. H. 1976, A\&A, 47, 221

Leinert, C., et al. 1998, A\&AS, 127, 1

Levasseur-Regourd, A. C., \& Dumont, R. 1980, A\&A, 84, 277

Low, F. J., Young, E., Beintema, D. A., et al. 1984, ApJ, 278, L19

Lumme, K. \& Bowell, E. 1985, Icarus, 62, 54

Min, M., Kama, M., Dominik, C., \& Waters, L. B. F. M. 2010, A\&A, 509, L6

Monet, D. G., et al. 2003, AJ, 125, 984

Muinonen, K., Piironen, J., Shkuratov, Y. G., Ovcharenko, A., \& Clark, B. E. 2002, Asteroids III, 123

Mukai, T., Fujino, M., Ishiguro, M., Nakamura, R., Ueno, M., Usui, F., \& Kwon, S. M. 2003, Icarus, 162, 337

Nesvorný, D., Bottke, W. F., Levison, H. F., \& Dones, L. 2003, ApJ, 591, 486

Nesvorný, D., Vokrouhlický, D., Bottke, W. F., \& Sykes, M. 2006, Icarus, 181, 107

Nesvorný, D., Jenniskens, P., Levison, H. F., Bottke, W. F., Vokrouhlický, D., \& Gounelle, M. 2010, ApJ, 713, 816

Reach, W. T., Franz, B. A., \& Weiland, J. L. 1997, Icarus, 127, 461

Renard, J. B., Levasseur-Regourd, A. C., \& Dumont, R. 1995, A\&A, 304, 602 
Roosen, R. G. 1970, Icarus, 13, 184

Roosen, R. G. 1970, Icarus, 13, 523

Snodgrass, C., Tubiana, C., Vincent, J.-B., et al. 2010, Nature, 467, 814

Sykes, M. V. 1990, Icarus, 85, 267

Sykes, M. V., \& Greenberg, R. 1986, Icarus, 65, 51

Tanabe, H. 1965, PASJ, 17, 339

Tsumura, K., Battle, J., Bock, J., et al. 2010, ApJ, 719, 394

Ueno, M., et al. 2007, Dust in Planetary Systems, 643, 197

Ueno, M., et al. 2005, LPI Contributions, 1280, 156

Usui, F., et al. 2002, 8th Asian-Pacific Regional Meeting, Volume II, 231

Usui, F., Kuroda, D., Müller, T. G., et al. 2011, PASJ, 63, 1117

Weinberg, J. L., Hanner, M. S., Mann, H. M., Hutchison, P. B., \& Fimmel, R. 1973, Space Res., 1187

Wolstencroft, R. D., \& Rose, L. J. 1967, ApJ, 147, 271

Zacharias, N., Rafferty, T. J., \& Zacharias, M. I. 2000, Astronomical Data Analysis Software and Systems IX, 216, 427 

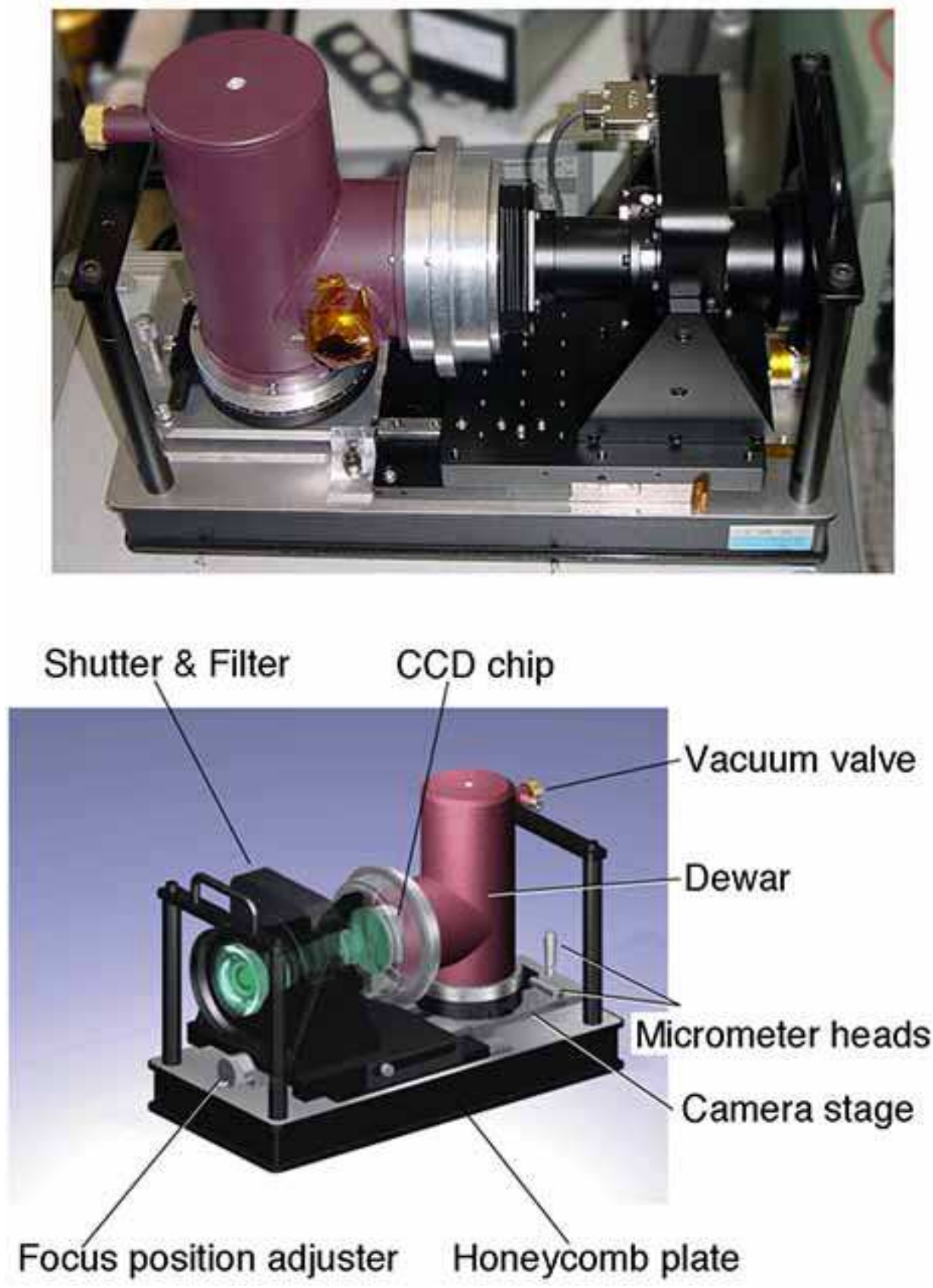

Fig. 1.- Photograph (top) and schematic view (bottom) of WIZARD. It consists of a cryogenic dewar and optics. These two units are connected with a bellows tube and mounted on a thick honeycomb plate. The mechanical shutter and glass filter are placed at the pupil position. The system is mounted on an equatorial mounting for star-tracking. 


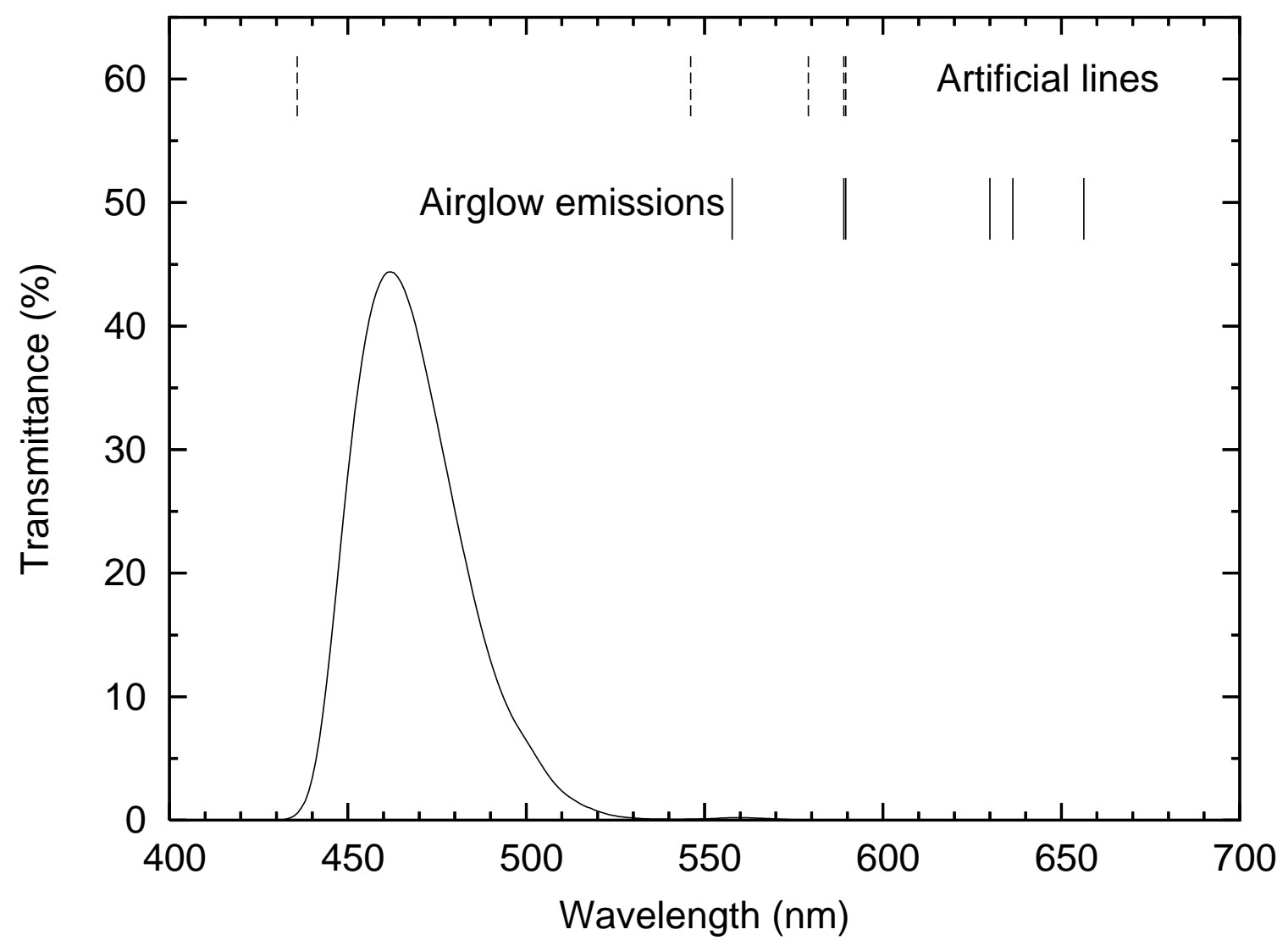

Fig. 2. - Transmittance of the filter we applied for the Gegenschein and zodiacal light observations. This filter is designed to avoid the prominent natural emissions and artificial emissions, indicated by solid lines and dashed lines, respectively. They consist of O I (557.7, 630.0, and $636.4 \mathrm{~nm}), \mathrm{NaI}$ (589.0 and $589.6 \mathrm{~nm})$, and $\mathrm{H} \alpha(656.3 \mathrm{~nm})$ for the natural emissions and $\mathrm{HgI}(435.8,546.1$, and $579.1 \mathrm{~nm})$ and $\mathrm{NaI}(589.0$ and $589.6 \mathrm{~nm})$ for the artificial emissions, respectively. 


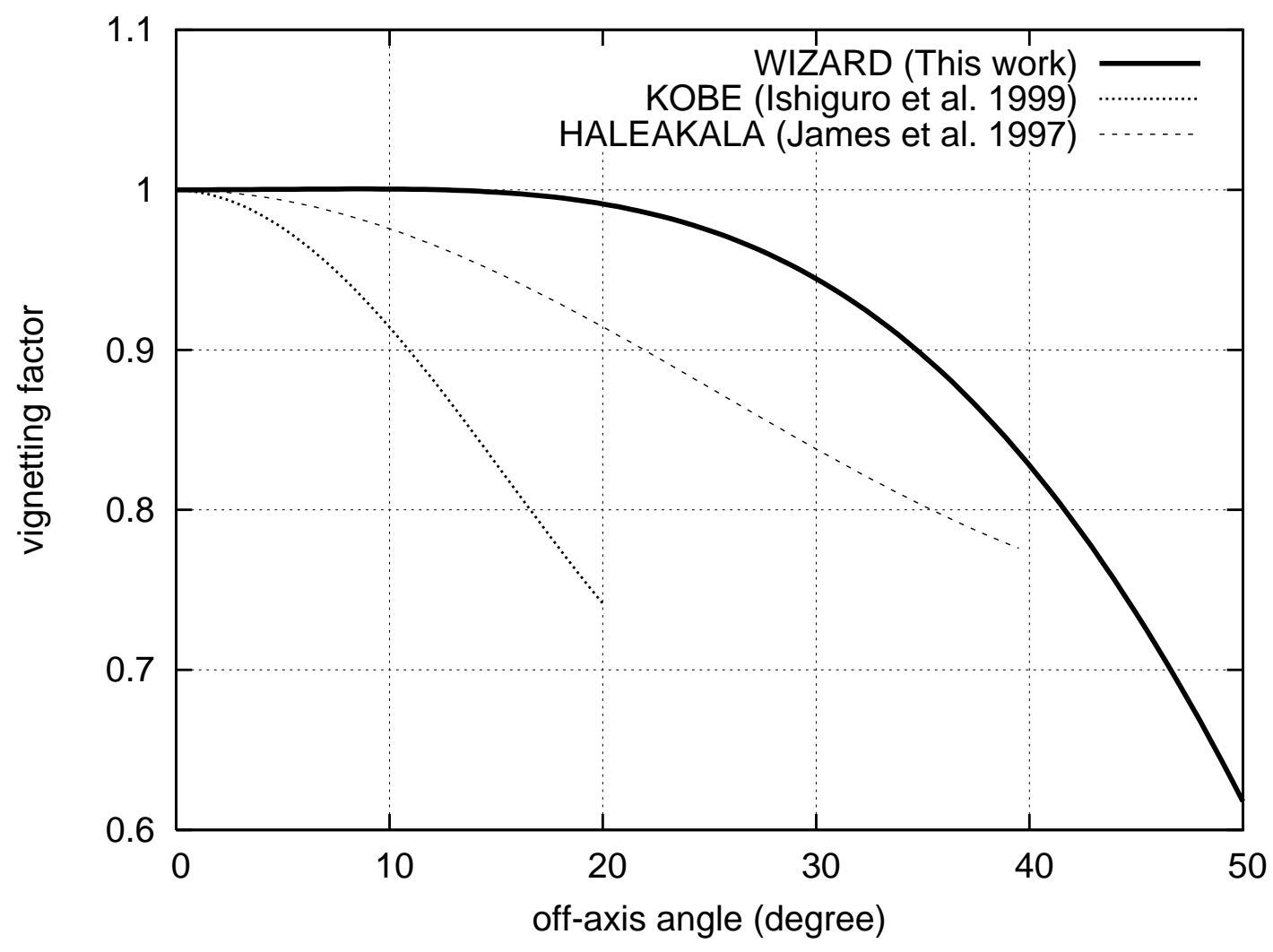

Fig. 3. - Vignetting factor of WIZARD normalized at the optical center (thick solid line). Two vignetting functions in Ishiguro et al. (1999a) and James et al. (1997) are also drawn for comparison. It is clear that WIZARD optics has little vignetting within $20^{\circ}$ from the center. 


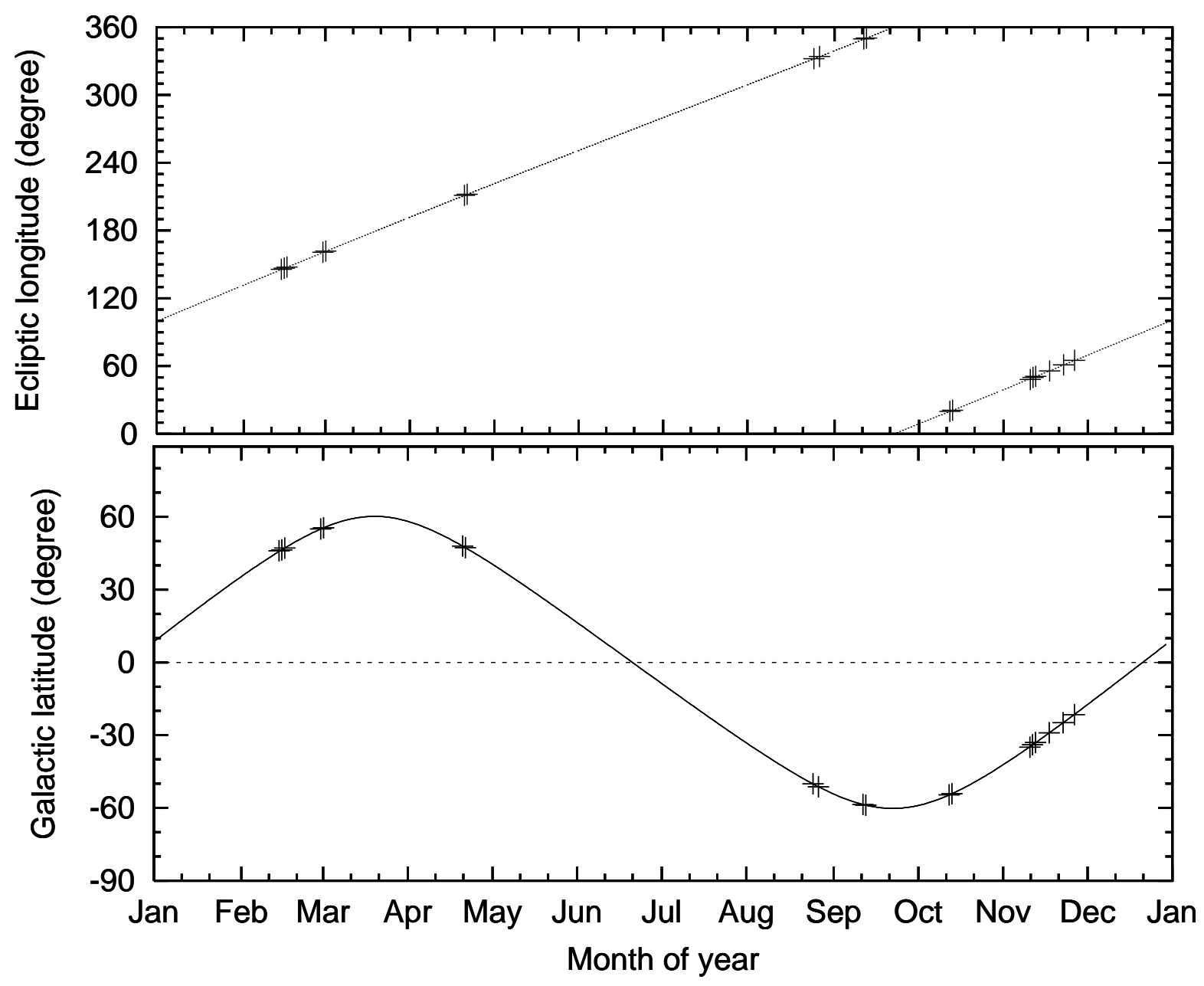

Fig. 4.- Ecliptic longitude (top) and Galactic latitude (bottom) of the antisolar point as a function of the day of the year. Crosses denote the ecliptic longitude and Galactic latitude when we made the observations. The antisolar point lies on the Galactic plane on June 21 and December 22. 


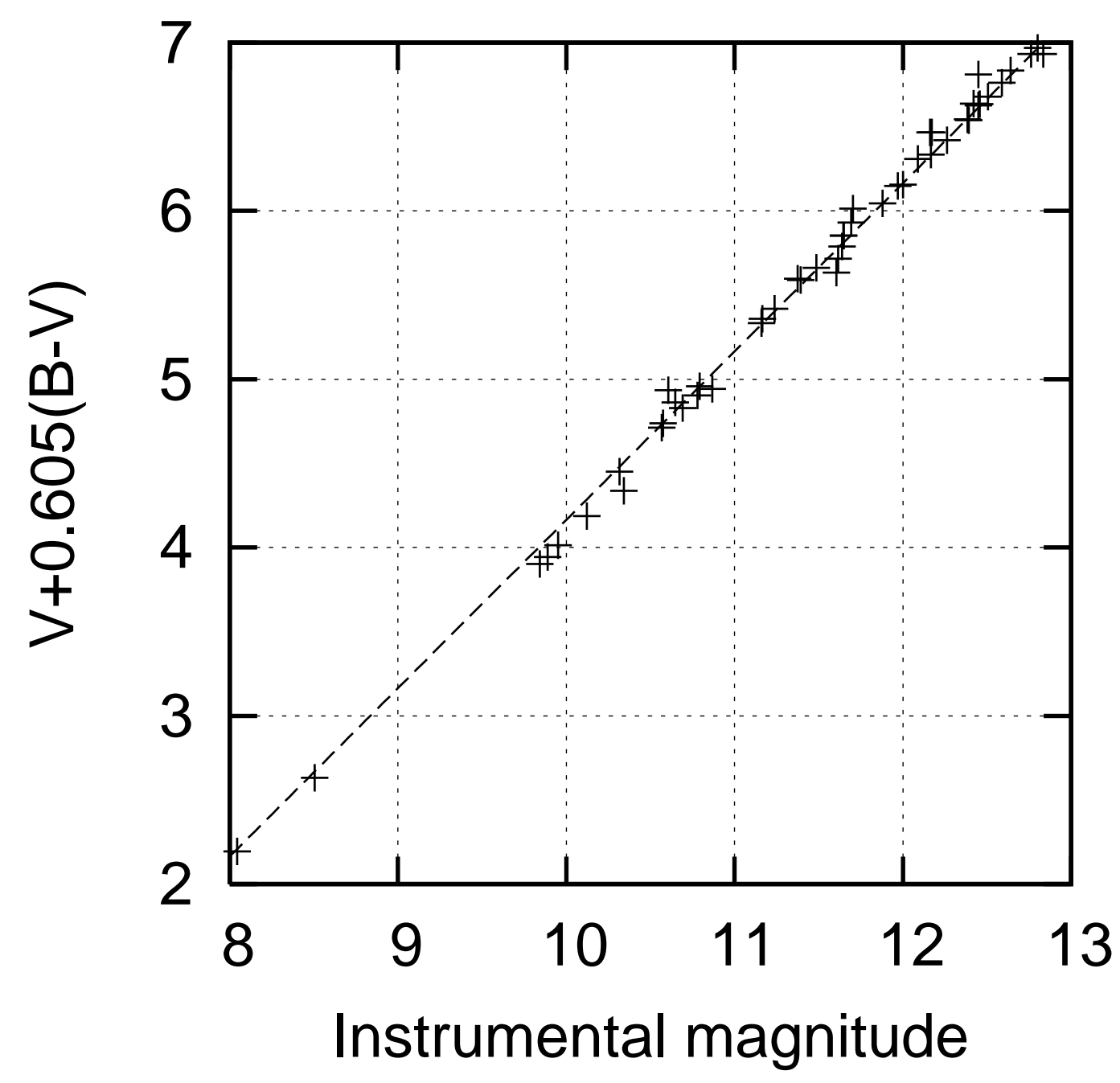

Fig. 5.- Comparison between the instrumental magnitude defined as $-2.5 \log (\mathrm{DN})+$ constant and color-corrected catalog magnitude of field stars, where $D N$ denotes CCD count. Since these stars exist near the zenith (within an airmass of 1.07), we did not correct for the zenith extinction term (see Section 2.2). 


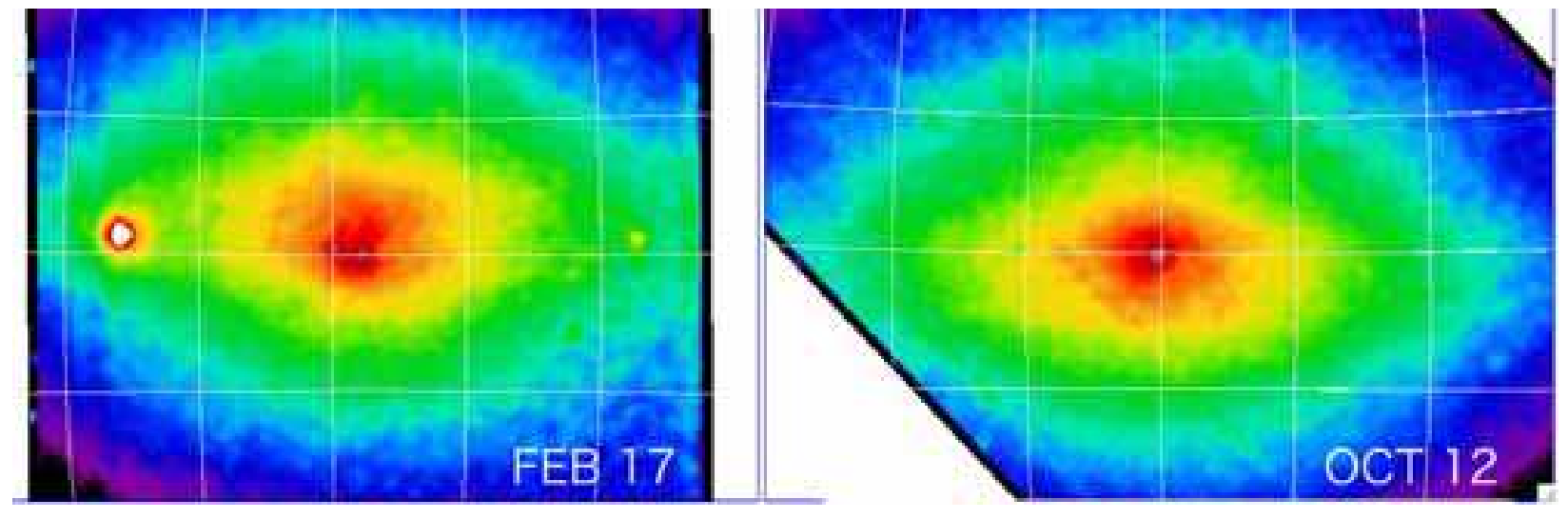

Fig. 6.- Contour maps of the Gegenschein taken on (left) February 17 and (right) October 12. The interval of grid is $10^{\circ}$. 


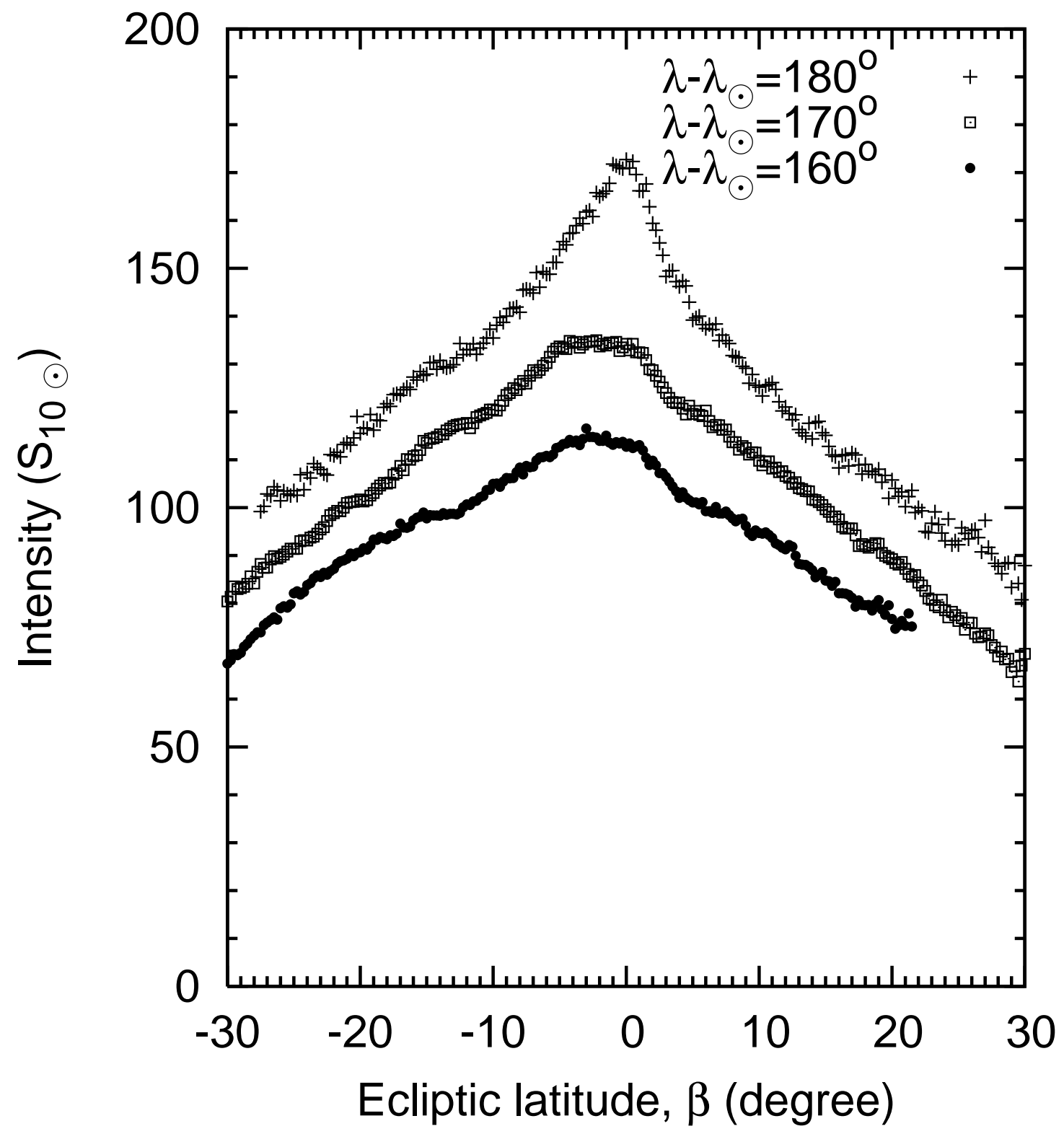

Fig. 7. - Surface brightness profile along the ecliptic latitude at $\lambda-\lambda_{\odot}=180^{\circ}$ (top), $170^{\circ}($ middle $)$, and $160^{\circ}$ (bottom). For clarity, the middle and lower profiles are shifted by $10 \mathrm{~S}_{\odot 10}$ and $20 \mathrm{~S}_{\odot 10}$, respectively. 

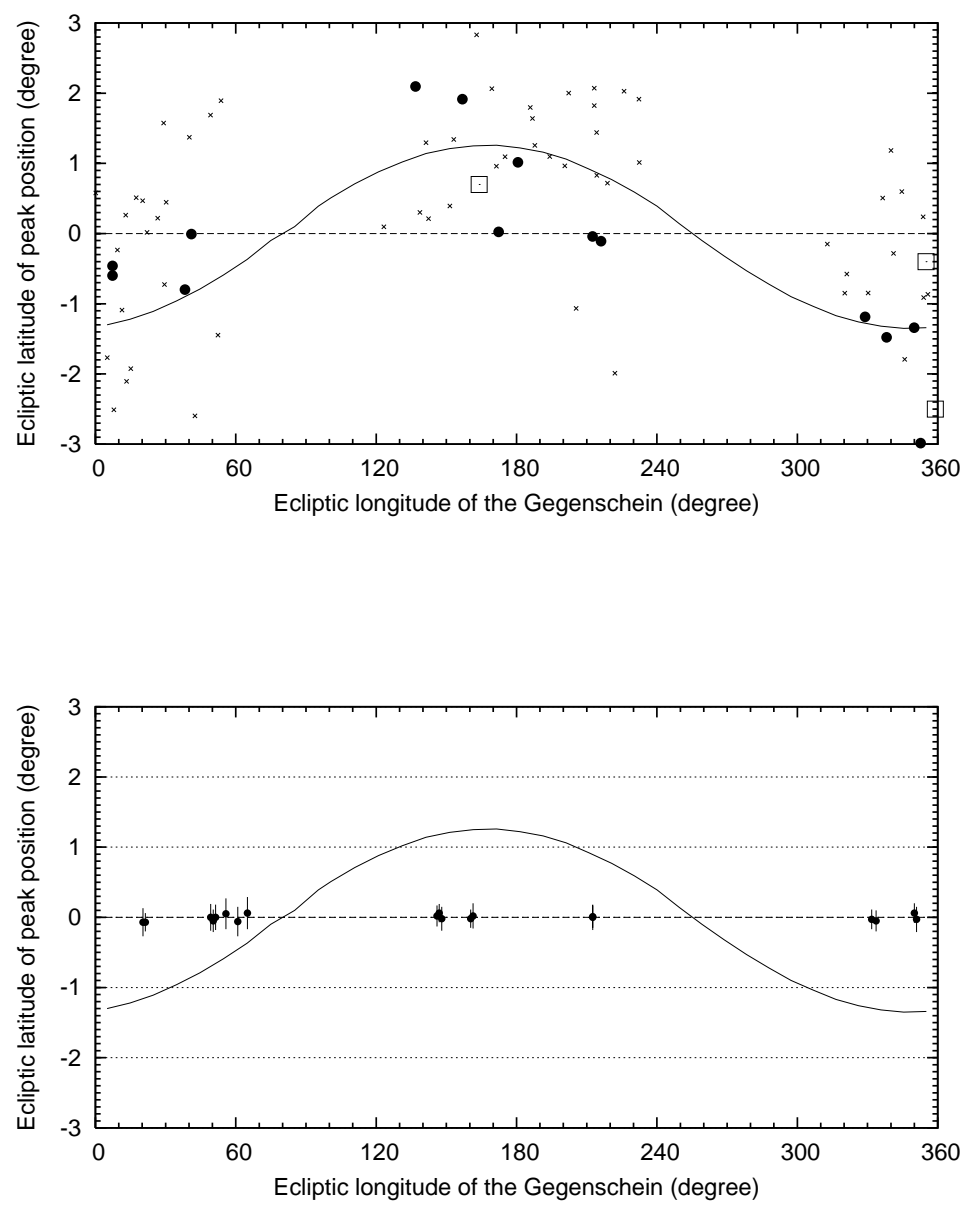

Fig. 8.- Observed latitude of the maximum intensity of the Gegenschein. In the top panel, the data are from photographic (crosses), photoelectric (filled circles), and CCD observations (open squares) (Roosen 1970b; Ishiguro et al. 1998). Our results are shown in the bottom panel. For comparison, we show the position predicted using a plausible model on the basis of Mukai et al. (2003) and Kwon et al. (2004) (solid line). In the model, the plane of symmetry for $i_{\mathrm{sym}}=2^{\circ}$ and $\Omega_{\text {sym }}=80^{\circ}$ is considered. The scattering phase function in Hong (1985) was applied for the model, assuming $\nu=1.3$. 


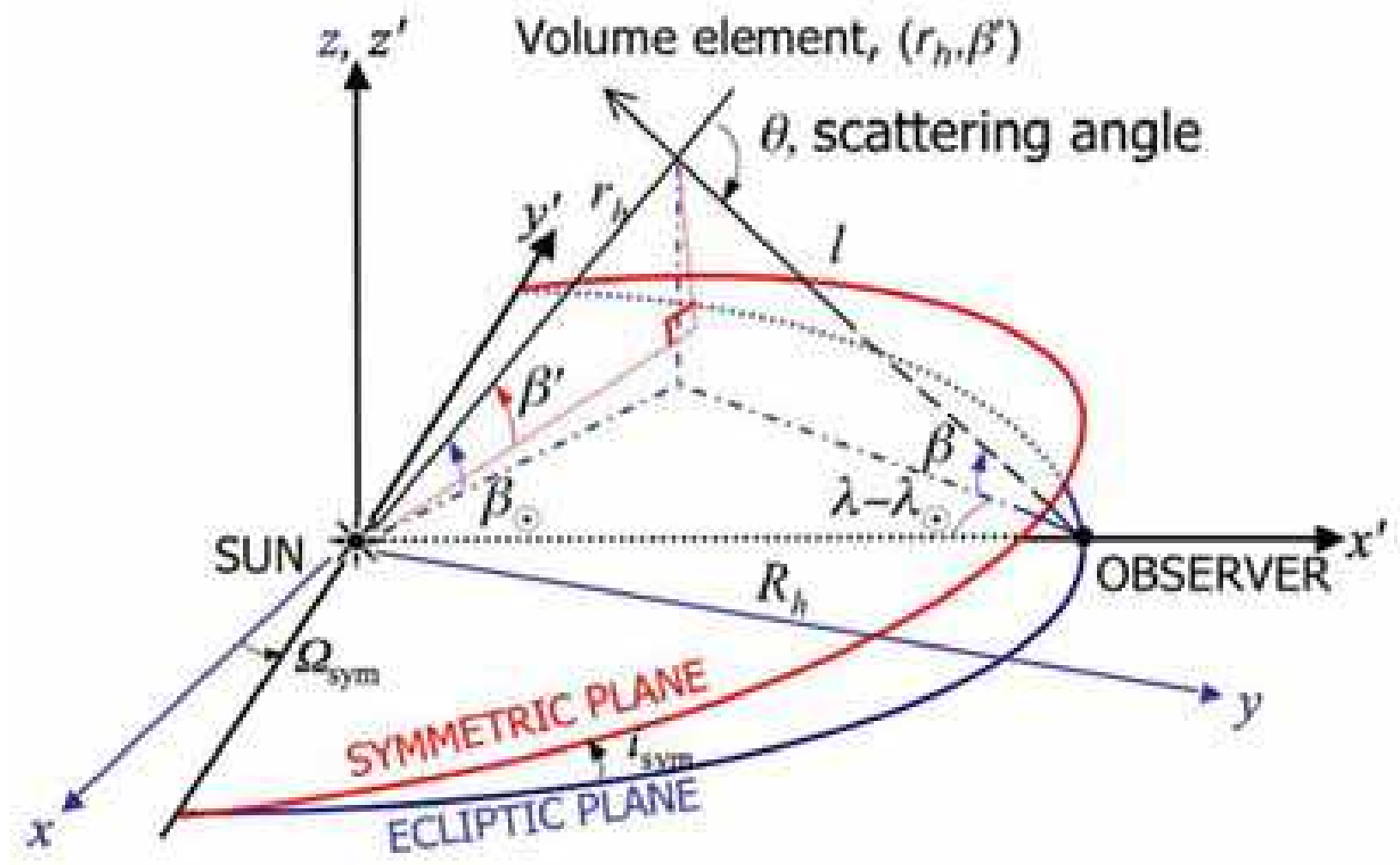

Fig. 9. - The viewing geometry for light scattered by a dust volume element. The scattering angle $\theta$ is measured from the line of sight to the antisolar direction at the volume element. 

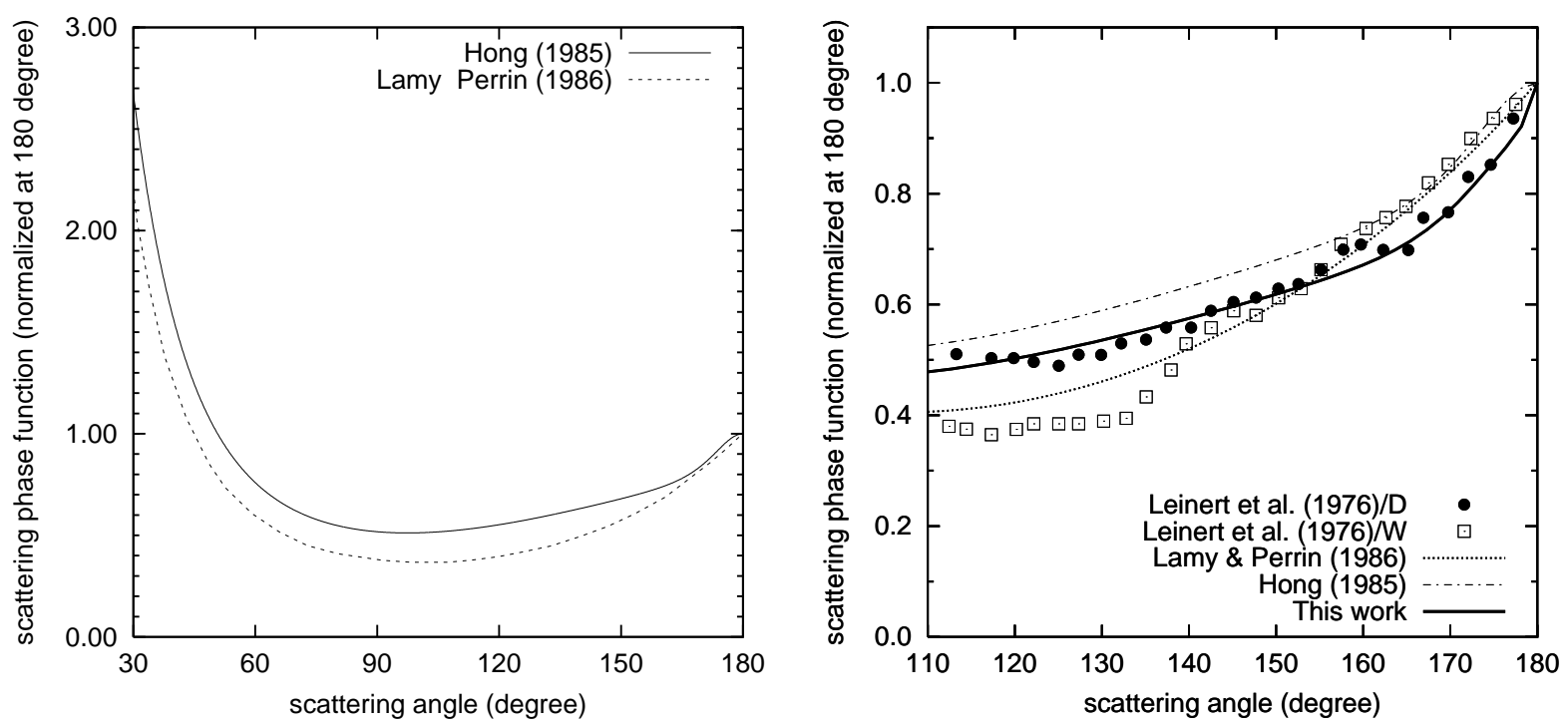

Fig. 10.- (Left) The scattering phase functions from Hong (1985) and Lamy \& Perrin (1986) for a radial power law $\nu=1$. (Right) Comparison between scattering phase functions obtained by other authors and us. 

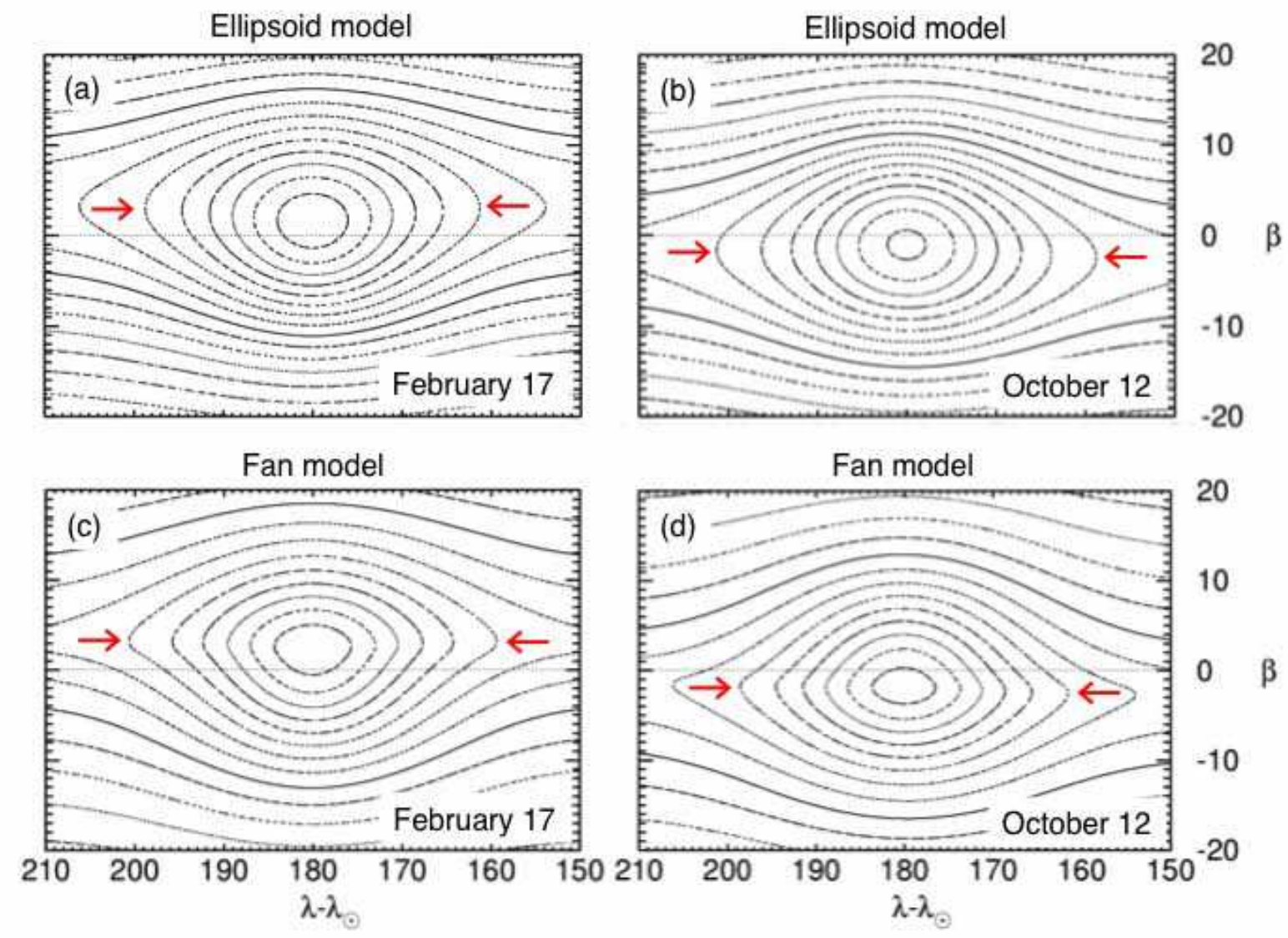

Fig. 11.- An isophoto map, in arbitrary units, of the Gegenschein at two different days of year based on the ellipsoid model (top) and the fan model (bottom). The scattering phase function of Hong (1985) was applied. 

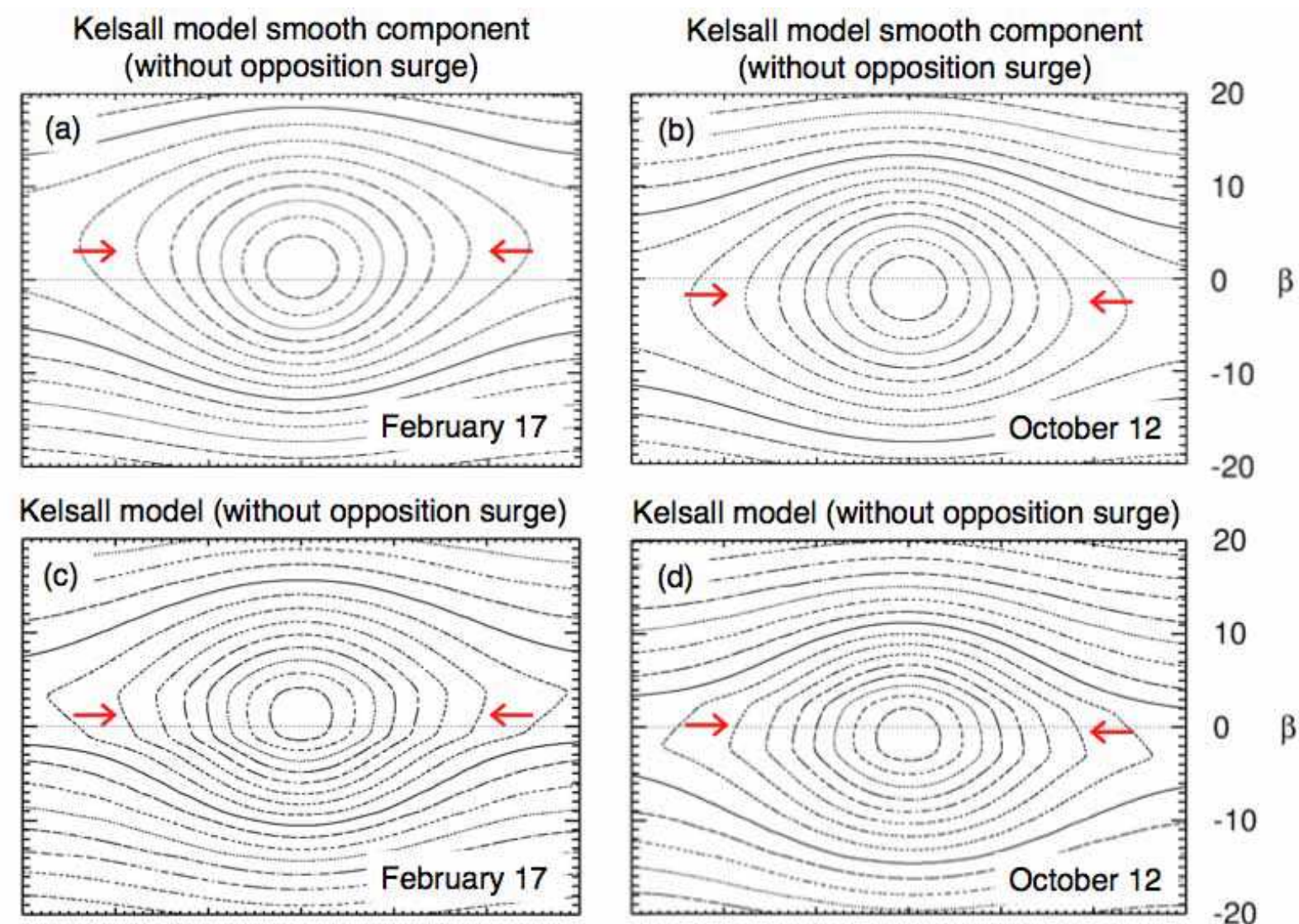

$-20$
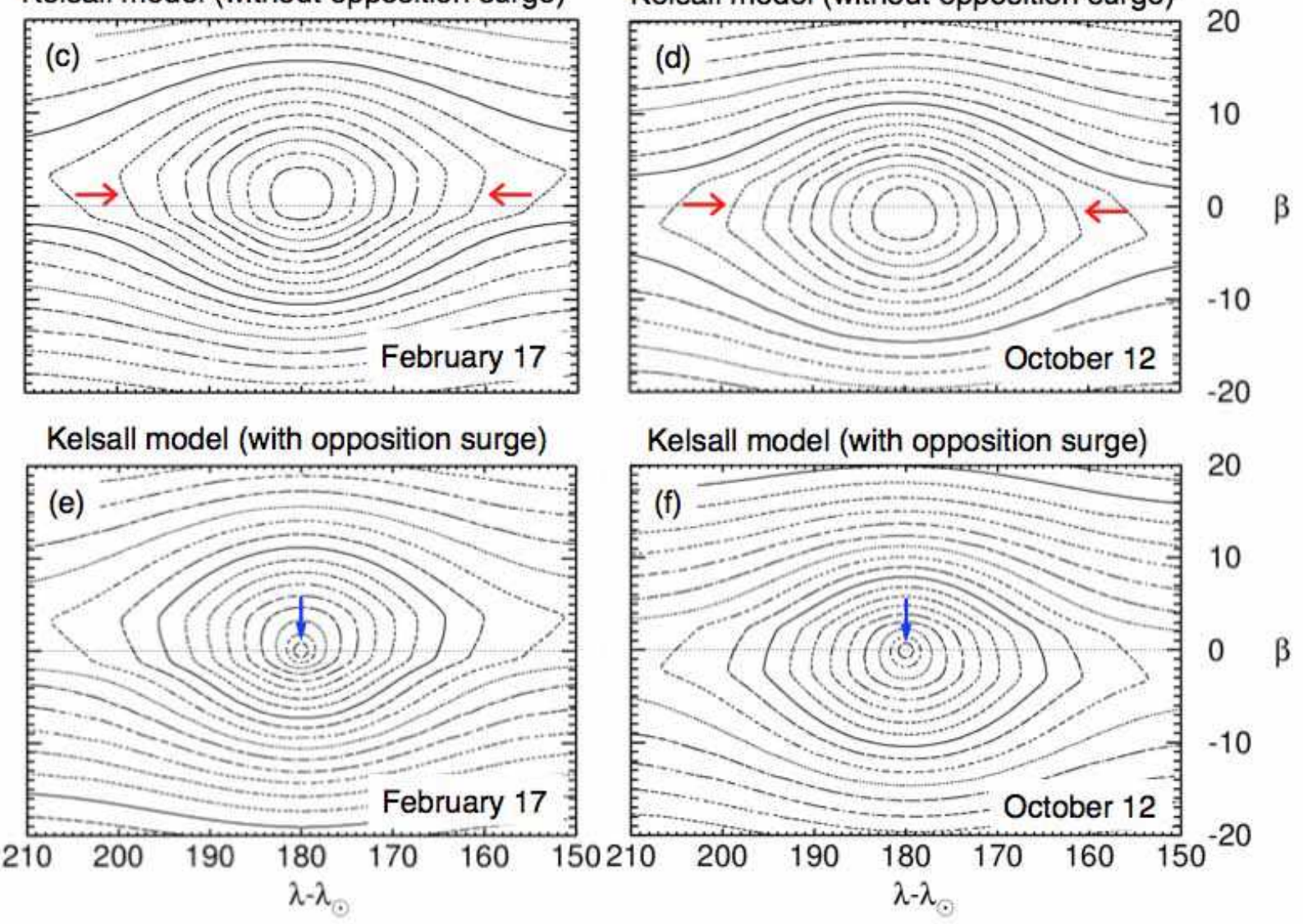

Fig. 12.- An isophoto map, in arbitrary units, of the Gegenschein at days from two different seasons based on the infrared 3-D model (Kelsall et al. 1998). We applied only the smooth component in the top panels and all components including dust bands in the middle and bottom panels. The original scattering phase function of Hong (1985) was applied in the top and middle panels, while the modified scattering phase function was applied in the bottom two panels. 

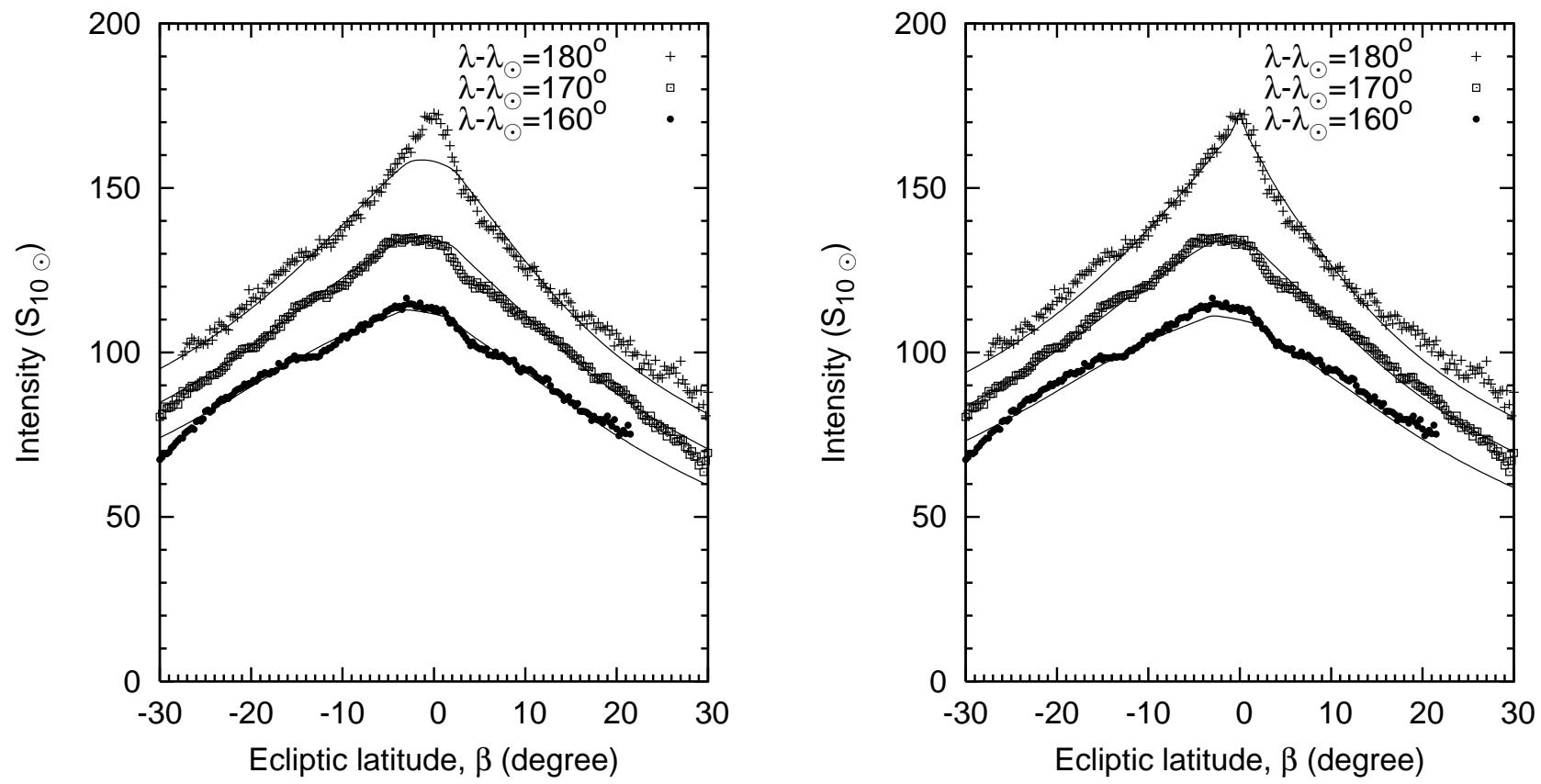

Fig. 13. - Comparison between the observed brightness profiles with those obtained from the model (solid lines). We applied the original scattering phase function of Hong (1985) assuming $\nu=1.3$ in the left panel and a modified scattering phase function of Hong (1985) with $15 \%$ enhancement at opposition in the right panel. 

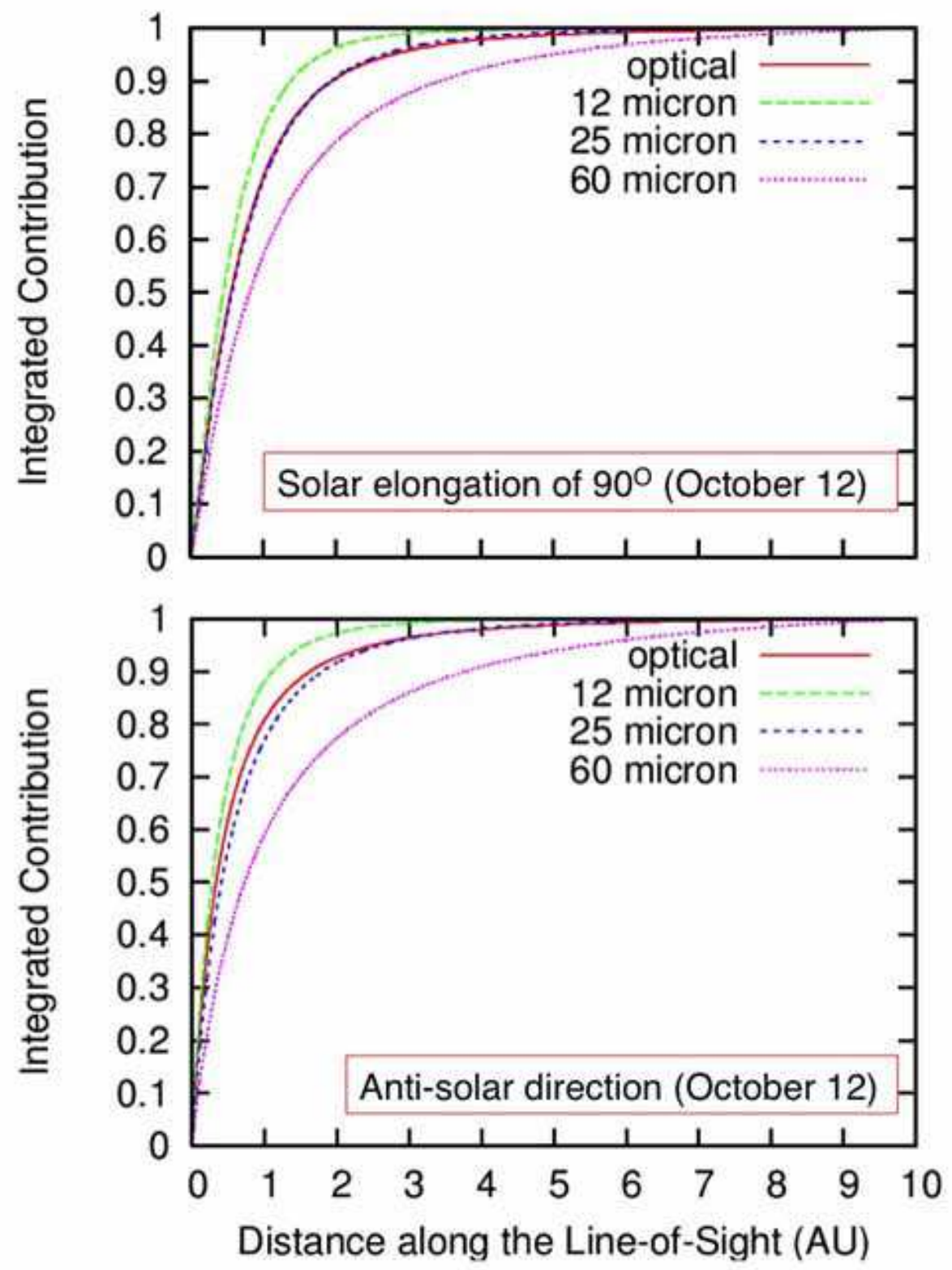

Fig. 14. - The line of sight depth of optical zodiacal light and infrared zodiacal emission at at $\lambda-\lambda_{\odot}=90^{\circ}$ and $\lambda-\lambda_{\odot}=180^{\circ}$ on the ecliptic plane. 
Table 1: Summary of the WIZARD performance

\begin{tabular}{|c|c|}
\hline Field-of-view & $49^{\circ} \times 98^{\circ}$ \\
\hline Pixel resolution & $1.435^{\prime}$ \\
\hline Gain factor & $0.825\left(\mathrm{e}^{-} / \mathrm{ADU}\right)$ \\
\hline Readout noise & $20-25\left(\mathrm{e}^{-}\right)$ \\
\hline Stability of the CCD zero level & $\lesssim 0.6 \%$ of the Gegenschein brightness \\
\hline Vignetting & $30 \%$ (at $47^{\circ}$ from the optical center) \\
\hline Pixel-to-pixel responsivity variation & $\lesssim 0.3 \%$ \\
\hline
\end{tabular}


Table 2. Observation summary

\begin{tabular}{|c|c|c|c|c|c|c|c|}
\hline & Date & $\mathrm{UT}^{a}$ & $r_{h}^{b}$ & $\lambda_{\oplus}^{c}$ & $Z^{d}$ & $l^{e}$ & $b^{f}$ \\
\hline \multirow[t]{11}{*}{2003} & \multirow[t]{2}{*}{ March 01} & $10: 05$ & 0.991 & 160.36 & 14.04 & 241 & +55 \\
\hline & & $10: 29$ & 0.991 & 160.37 & 12.21 & 241 & +55 \\
\hline & \multirow[t]{4}{*}{ March 02} & $09: 35$ & 0.991 & 161.34 & 18.99 & 242 & +55 \\
\hline & & $09: 58$ & 0.991 & 161.36 & 15.25 & 242 & +55 \\
\hline & & $10: 45$ & 0.991 & 161.39 & 12.82 & 242 & +55 \\
\hline & & $11: 35$ & 0.991 & 161.42 & 19.41 & 242 & +55 \\
\hline & \multirow[t]{3}{*}{ August 25} & 09:58 & 1.011 & 331.75 & 31.33 & 49 & -50 \\
\hline & & $10: 26$ & 1.011 & 331.77 & 30.68 & 49 & -50 \\
\hline & & $10: 49$ & 1.011 & 331.78 & 31.28 & 49 & -50 \\
\hline & \multirow[t]{2}{*}{ August 27} & 10:06 & 1.010 & 333.69 & 30.28 & 52 & -51 \\
\hline & & $10: 29$ & 1.010 & 333.70 & 30.01 & 52 & -51 \\
\hline \multirow[t]{22}{*}{2004} & \multirow[t]{2}{*}{ February 15} & 09:41 & 0.988 & 145.98 & 14.87 & 223 & +46 \\
\hline & & 10:00 & 0.988 & 145.99 & 11.06 & 223 & +46 \\
\hline & \multirow[t]{4}{*}{ February 16} & $08: 23$ & 0.988 & 146.94 & 32.64 & 224 & +47 \\
\hline & & 09:01 & 0.988 & 146.96 & 23.86 & 224 & +47 \\
\hline & & 09:41 & 0.988 & 146.99 & 15.03 & 224 & +47 \\
\hline & & $10: 05$ & 0.988 & 147.01 & 10.39 & 224 & +47 \\
\hline & \multirow[t]{3}{*}{ February 17} & 09:09 & 0.988 & 147.98 & 22.17 & 225 & +47 \\
\hline & & $09: 27$ & 0.988 & 147.99 & 18.18 & 225 & +47 \\
\hline & & $09: 44$ & 0.988 & 148.00 & 14.58 & 225 & +47 \\
\hline & \multirow[t]{4}{*}{ April 21} & $09: 28$ & 1.005 & 211.55 & 34.34 & 327 & +48 \\
\hline & & $10: 05$ & 1.005 & 211.58 & 32.07 & 327 & +48 \\
\hline & & $10: 24$ & 1.005 & 211.59 & 31.87 & 327 & +48 \\
\hline & & $10: 42$ & 1.005 & 211.60 & 32.30 & 327 & +48 \\
\hline & \multirow[t]{2}{*}{ April 22} & $09: 24$ & 1.005 & 212.52 & 35.01 & 328 & +47 \\
\hline & & 09:42 & 1.005 & 212.54 & 33.52 & 328 & +47 \\
\hline & \multirow[t]{2}{*}{ September 12} & $11: 53$ & 1.006 & 349.98 & 33.37 & 77 & -59 \\
\hline & & $12: 12$ & 1.006 & 350.00 & 36.79 & 77 & -59 \\
\hline & \multirow[t]{3}{*}{ September 13} & $11: 25$ & 1.006 & 350.94 & 28.71 & 79 & -59 \\
\hline & & $11: 45$ & 1.006 & 350.95 & 31.80 & 79 & -59 \\
\hline & & 12:04 & 1.006 & 350.96 & 35.12 & 79 & -59 \\
\hline & \multirow[t]{2}{*}{ October 13} & $08: 52$ & 0.998 & 20.29 & 21.87 & 133 & -54 \\
\hline & & $09: 13$ & 0.998 & 20.31 & 17.82 & 133 & -54 \\
\hline
\end{tabular}


Table 2-Continued

\begin{tabular}{ccccccc}
\hline \hline Date & $\mathrm{UT}^{a}$ & $r_{h}{ }^{b}$ & $\lambda_{\oplus}{ }^{c}$ & $Z^{d}$ & $l^{e}$ & $b^{f}$ \\
\hline \multirow{2}{*}{ October 14 } & $09: 22$ & 0.997 & 21.30 & 15.95 & 134 & -54 \\
& $09: 41$ & 0.997 & 21.32 & 13.19 & 134 & -54 \\
& $09: 57$ & 0.997 & 21.33 & 11.79 & 134 & -54 \\
& $10: 13$ & 0.997 & 21.34 & 11.58 & 135 & -54 \\
November 11 & $09: 59$ & 0.990 & 49.29 & 2.77 & 163 & -34 \\
& $10: 18$ & 0.990 & 49.30 & 3.70 & 163 & -34 \\
& $10: 36$ & 0.990 & 49.31 & 7.53 & 163 & -34 \\
November 12 & $10: 16$ & 0.990 & 50.30 & 3.14 & 164 & -34 \\
& $10: 33$ & 0.990 & 50.32 & 6.74 & 164 & -34 \\
& $10: 49$ & 0.990 & 50.33 & 10.41 & 164 & -34 \\
November 13 & $10: 19$ & 0.989 & 51.31 & 3.54 & 165 & -33 \\
& $10: 52$ & 0.989 & 51.34 & 11.02 & 165 & -33 \\
November 18 & $08: 47$ & 0.988 & 55.77 & 18.76 & 168 & -29 \\
& $09: 05$ & 0.988 & 55.78 & 14.53 & 168 & -29 \\
November 23 & $08: 41$ & 0.987 & 60.81 & 20.38 & 171 & -25 \\
& $08: 59$ & 0.987 & 60.83 & 16.16 & 171 & -25 \\
November 27 & $12: 01$ & 0.987 & 65.00 & 26.21 & 173 & -22 \\
\hline
\end{tabular}

Note. - ${ }^{a}$ Median time of exposure.

${ }^{b}$ Heliocentric distance of the Earth in AU.

${ }^{c}$ Ecliptic longitude of the antisolar point with the equinox of J2000 in degrees.

$d$ Zenith distance of the antisolar point in degrees.

${ }^{e}$ Galactic longitude of the antisolar point in degrees.

${ }^{f}$ Galactic latitude of the antisolar point in degrees. 